\title{
Targeting enhancer reprogramming to mitigate MEK inhibitor resistance in preclinical models of advanced ovarian cancer
}

\author{
Shini Liu, ${ }^{1}$ Qiong Zou, ${ }^{1}$ Jie-Ping Chen, ${ }^{1}$ Xiaosai Yao, ${ }^{2}$ Peiyong Guan, ${ }^{3}$ Weiting Liang, ${ }^{1}$ Peng Deng, ${ }^{2}$ Xiaowei Lai, Jiaxin Yin, ${ }^{1}$ \\ Jinghong Chen, ${ }^{1}$ Rui Chen, ${ }^{1}$ Zhaoliang Yu, ${ }^{4}$ Rong Xiao, ${ }^{1}$ Yichen Sun, ${ }^{1}$ Jing Han Hong, ${ }^{3}$ Hui Liu, ${ }^{1}$ Huaiwu Lu, Jianfeng Chen, ${ }^{1}$ \\ Jin-Xin Bei, ${ }^{1}$ Joanna Koh, ${ }^{6}$ Jason Yongsheng Chan, ${ }^{6}$ Baohua Wang, ${ }^{7}$ Tiebang Kang, ${ }^{1}$ Qiang Yu, ${ }^{3,8}$ Bin-Tean Teh, ${ }^{2,3,6,9,10}$ \\ Jihong Liu, ${ }^{1}$ Ying Xiong, ${ }^{1}$ and Jing Tan ${ }^{1,6,11}$
}

'Sun Yat-sen University Cancer Center, State Key Laboratory of Oncology in South China, Collaborative Innovation Center for Cancer Medicine, Guangzhou, Guangdong, China. Institute of Molecular and Cell Biology, Singapore. ${ }^{3}$ Cancer and Stem Cell Biology Program, Duke-NUS Medical School, Singapore. ${ }^{4}$ Cuangdong Provincial Key Laboratory of Colorectal and Pelvic Floor Diseases, The Sixth Affiliated Hospital of Sun Yat-sen University, Guangzhou, Guangdong, China. ${ }^{5}$ Sun Yat-sen Memorial Hospital of Sun Yat-sen University, Guangzhou, Guangdong, China. 'Laboratory of Cancer Epigenome, Division of Medical Sciences, National Cancer Centre Singapore, Singapore. ${ }^{7}$ The First Hospital of Guangzhou University of Chinese Medicine, Guangzhou, Guangdong, China. ${ }^{8}$ Cenome Institute of Singapore, Agency for Science, Technology and Research (A*STAR), Singapore. ${ }^{9}$ Cancer Science Institute of Singapore, National University of Singapore, Singapore. ${ }^{10}$ SingHealth Duke-NUS Institute of Precision Medicine, National Heart Centre Singapore, Singapore. "Affiliated Cancer Hospital and Institute of Guangzhou Medical University, Guangzhou, Guangdong, China.

\begin{abstract}
Ovarian cancer is characterized by aberrant activation of the mitogen-activated protein kinase (MAPK), highlighting the importance of targeting the MAPK pathway as an attractive therapeutic strategy. However, the clinical efficacy of MEK inhibitors is limited by intrinsic or acquired drug resistance. Here, we established patient-derived ovarian cancer models resistant to MEK inhibitors and demonstrated that resistance to the clinically approved MEK inhibitor trametinib was associated with enhancer reprogramming. We also showed that enhancer decommissioning induced the downregulation of negative regulators of the MAPK pathway, leading to constitutive ERK activation and acquired resistance to trametinib. Epigenetic compound screening uncovered that HDAC inhibitors could alter the enhancer reprogramming and upregulate the expression of MAPK negative regulators, resulting in sustained MAPK inhibition and reversal of trametinib resistance. Consequently, a combination of HDAC inhibitor and trametinib demonstrated a synergistic antitumor effect in vitro and in vivo, including patient-derived xenograft mouse models. These findings demonstrated that enhancer reprogramming of the MAPK regulatory pathway might serve as a potential mechanism underlying MAPK inhibitor resistance and concurrent targeting of epigenetic pathways and MAPK signaling might provide an effective treatment strategy for advanced ovarian cancer.
\end{abstract}

\section{Introduction}

Epithelial ovarian cancer (OV) is the leading cause of mortality from gynecological malignancies (1); the overall survival rate has remained poor at $30 \%$ to $50 \%$ for decades, mainly owing to late diagnosis and resistance to first-line chemotherapy (2-4). Therefore, there is an unmet clinical demand to discover novel strategies, particularly targeted therapies, to treat advanced OV. Mitogen-activated protein kinase (MAPK) signaling is one of the most deregulated oncogenic pathways across a variety of human cancers, including colon cancer and OV (5-7). Integrative network analysis of The Cancer Genome Atlas data for OV revealed that approximately $30 \%$ of patients with OV harbor aberrant activated MAPK oncogenic signaling, which involves inactivation of the RAS GTPase-activating protein neurofibromatosis 1 (NF1) and

Authorship note: SL, QZ, and Jieping Chen contributed equally to this work Conflict of interest: The authors have declared that no conflict of interest exists. Copyright: ( 2021, American Society for Clinical Investigation.

Submitted: October 12, 2020; Accepted: August 24, 2021; Published: October 15, 2021 Reference information: J Clin Invest. 2021;131(20):e145035.

https://doi.org/10.1172/JCl145035. activation of $R A S / B R A F$, suggesting that this pathway is a potential therapeutic target in OV treatment $(6,8)$.

MEK inhibitors, such as trametinib and selumetinib, have shown promising therapeutic efficacy and have been clinically approved for use in several tumor types characterized by BRAF activating mutations such as melanoma and non-small cell lung cancer $(9,10)$. MEK inhibitors have also demonstrated certain clinical activity in low-grade serous ovarian cancer (LGSOC) (11-13). Notably, clinical responses were recorded in both mutant and wildtype KRAS or BRAF tumors in a phase II clinical trial of the MEK inhibitor selumetinib in LGSOC (14), suggesting that MEK inhibition could also be effective in high-grade serous ovarian cancer (HGSOC). However, the potential utility of MEK inhibitors in highgrade serous subtypes has mainly been demonstrated through preclinical studies $(15,16)$ and isolated case reports $(17)$. In addition, the potential mechanisms and molecular predictors of outcome of MEK inhibitors are poorly investigated in preclinical studies, which hinders the implications of MEK inhibitors in OV therapy.

Despite the clinical effectiveness of MEK inhibitors, their clinical utilization has shown limited success owing to the rapid development of resistance $(18,19)$. To date, multiple resistance mech- 
anisms to MEK-targeted therapy have been elucidated, including gain of secondary target mutations, gene amplification, and compensatory activation of prosurvival signaling pathways. For instance, the acquisition of gain-of-function mutations in MEK1/2 has been found in colon cancer and melanoma, which abrogates MEK inhibitor binding, thereby maintaining ERK1/2 activity and resulting in acquired resistance to allosteric MEK inhibitor (20-22). Amplification of the upstream driving oncogenes such as $B R A F$ V600E and KRAS G13D has also been reported to account for MEK inhibitor resistance (23). Furthermore, adaptive kinome reprogramming of bypass signaling pathways, such as c-MET/ STAT3 and EGFR/HER3 signaling, has been shown to drive the occurrence of resistance $(24,25)$. In addition, activation of compensatory survival pathways has also been determined to result in drug resistance to MEK inhibitors in LGSOC and HGSOC (26). These findings highlighted the complexity of drug-resistance mechanisms in MEK-targeted therapy, which motivated us to systematically exploit the potential mechanisms of resistance to MEK inhibitors in robust preclinical models of $\mathrm{OV}$ to improve patient survival outcomes.

Here, we report the establishment of a series of patient-derived advanced OV models that were sensitive or resistant to trametinib, allowing for a robust platform to unravel mechanisms of drug resistance. Using integrative chromatin and transcriptome profiling, we demonstrated that enhancer reprogramming led to sustained activation of the MAPK pathway, which played a crucial role in MEK inhibitor resistance. We also showed that epigenetic drugs such as HDAC inhibitors could overcome this resistance, and a combination strategy targeting both MAPK signaling and HDAC could be required to maximize clinical benefit.

\section{Results}

In vitro effect of MEK inhibitor in OV models. We established 20 patient-derived advanced ovarian cancer cells (POVCs) as preclinical models to evaluate sensitivity to the MEK inhibitor trametinib. Half-maximal growth-inhibitory concentration $\left(\mathrm{IC}_{50}\right)$ analysis showed that approximately half of the POVCs were highly sensitive to trametinib at nanomolar concentrations. In contrast, the rest were relatively resistant, and 4 of them displayed complete resistance up to $10 \mu \mathrm{M}$ trametinib (Figure 1A). Similar findings were also observed in a panel of commercial OV cell lines (Figure 1B). The responses to MEK inhibition in both POVCs and the commercial cell lines were also confirmed with another MEK inhibitor (PD0325901) (Supplemental Figure 1, A and B; supplemental material available online with this article; https://oi.org/10.1172/JCI145035DS1). In addition, annexin V/propidium iodide assay indicated that trametinib remarkably increased the proportion of apoptotic cells in the sensitive cells but not in the resistant cells (Figure 1C). This inhibitory effect on growth for the cell was further confirmed by colony formation assays (Figure 1D). Immunoblot analysis showed that although the ERK phosphorylation was transiently inhibited by trametinib in both sensitive and resistant cells (Supplemental Figure 1C), it was reactivated in a time- and dose-dependent manner in resistant cells, but not in sensitive cells (Figure 1, E and F). Consistent with previous studies $(27,28)$, phosphorylated MEK (p-MEK) was dramatically increased upon trametinib treatment as a result of abrogation of a negative-feedback loop inhibiting the MAPK pathway in both sensi- tive and resistant cells. This observation raised a possibility that the sensitivity to trametinib could be associated with sustained inhibition of MAPK signaling, while the reactivation of MAPK signaling might lead to resistance to trametinib. To examine whether somatic alterations in the MAPK pathway were associated with sensitivity to MEK inhibitors, we examined the mutation status of MAPK pathway members, including $H / K / N-R A S, B R A F, M E K 1 / 2, E R K 1 / 2$, and $N F 1$, in the commercial OV cell lines using the Catalogue of Somatic Mutations in Cancer (COSMIC; ref. 29), the Broad Institute Cancer Cell Line Encyclopedia (CCLE; ref. 30), and other published databases $(31,32)$. The results showed that there was no significant correlation between the sensitivity to trametinib and the mutation status of MAPK pathway members (Supplemental Figure 1D), which was further confirmed by Fisher's exact test for the association between each single gene mutation and $\mathrm{IC}_{50}$ value of trametinib in these commercial cell lines (Supplemental Figure 1E). In addition, the baseline activity of p-ERK and p-MEK was not correlated with the sensitivity to trametinib in both patient-derived and commercial cells (Supplemental Figure 1, F and G). These observations were consistent with a previous study showing that the MEK inhibitor response was not significantly associated with mutation status or baseline levels of the phosphorylation status of the MAPK pathway (33). This suggested that alternative mechanisms such as epigenetic deregulation might be involved in the resistance to MEK inhibitors in OV.

Persistent ERK activation is associated with acquired resistance to $M E K$ inhibitor in $O V$. To investigate the molecular changes relevant to acquired resistance to MEK inhibitor, we chose 2 trametinib-sensitive OV cell lines (A2780 and OVCAR5) that harbor $B R A F$ or KRAS activating mutations and constitutively activated p-ERK signaling (Supplemental Figure 1, D and F) to establish the acquired resistance models, A2780-R and OVCAR5-R, through chronic exposure to trametinib. The chronically exposed cells were more resistant to trametinib and PD0325901 than the parental cells, as demonstrated by a significant rightward shift in the dose-response curve (Figure 2A and Supplemental Figure 2A) and further confirmed by colony formation (Figure 2B), as well as apoptosis assays (Figure 2C). Although trametinib was able to transiently inhibit ERK activity in both sensitive and resistant cells in a short period of time (Supplemental Figure 2B), ERK activity was sustained in resistant cells rather than sensitive cells in a dose-dependent manner (Figure 2, D and E). On the other hand, ERK was reactivated in resistant cells after prolonged culturing with trametinib (Figure 2, F and G), which was in agreement with the findings in Figure 1, E and F, suggesting that both intrinsic and acquired resistance was associated with ERK reactivation. To elucidate the molecular basis of ERK reactivation in acquired-resistant cell lines, we conducted whole exome sequencing in parental A2780 (A2780-P) and A2780-R cells. The mutational profiling analyses revealed that no additional mutations of the MAPK pathway members were found in resistant cells compared with parental cells (data not shown). This supports our hypothesis that epigenetic deregulation rather than genetic alterations could confer the resistance to MEK inhibitors in OV.

Enhancer reprogramming accompanies acquired resistance to $M E K$ inhibitors. To explore whether epigenetic dysregulation played a role in mediating the resistance to MEK inhibitors, we characterized the difference in chromatin remodeling between 


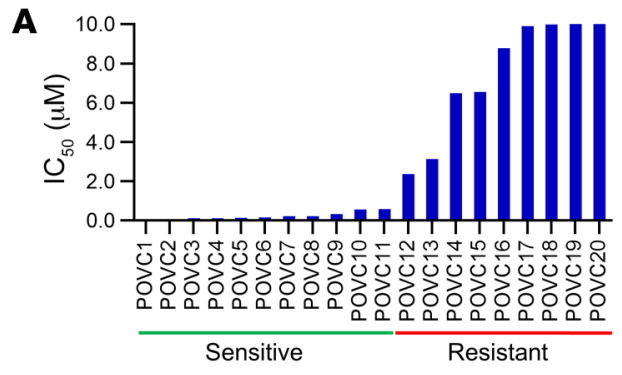

C

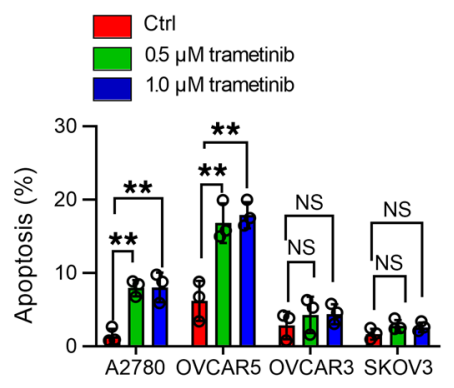

B

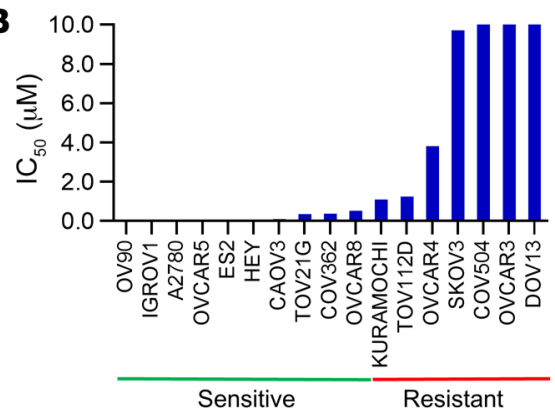

D

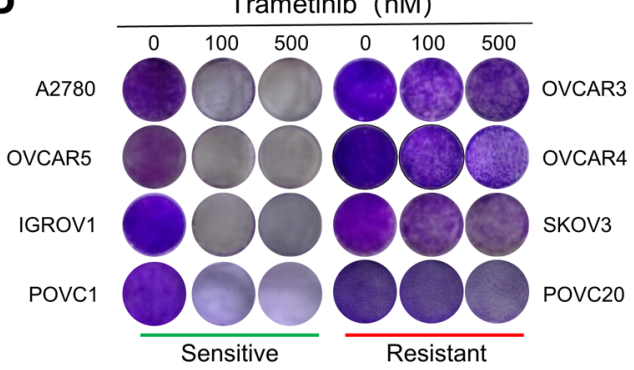

E

Trametinib (nM, $6 \mathrm{~h})$

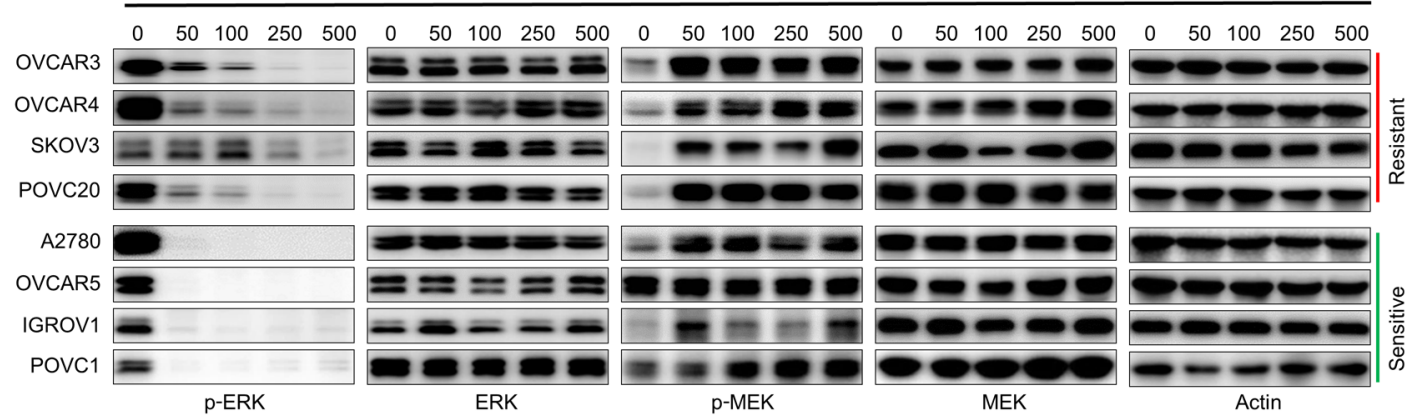

$\mathbf{F}$

Trametinib (100 nM, h)

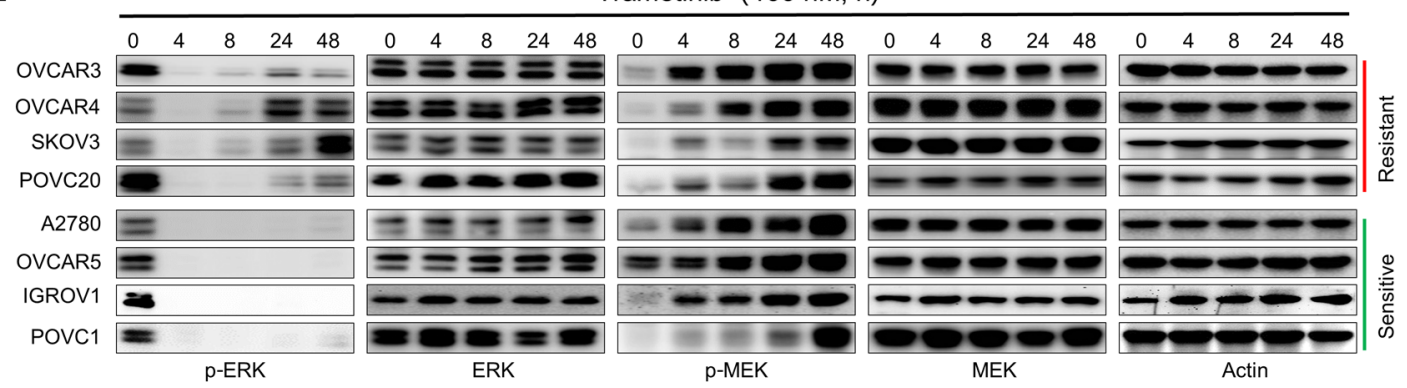

Figure 1. In vitro effect of MEK inhibitor in OV models. (A and B) Growth-inhibitory effect of trametinib on 20 patient-derived primary cells (A) and 17 commercial OV cell lines (B). Cells were treated with vehicle or different dosages of trametinib in a 4-day cell viability assay. The half-maximal inhibitory concentration $\left(\mathrm{IC}_{50}\right)$ values for trametinib are represented on the $y$ axis. Data represent the mean of 3 biological replicates. The cutoff value of IC $\mathrm{C}_{50}$ for sensitivity is $1.0 \mu \mathrm{M}$. (C) Quantification of the apoptotic cells in 2 sensitive cell lines (A2780 and OVCAR5) and 2 resistant cell lines (OVCAR3 and SKOV3) treated with vehicle or trametinib $(500 \mathrm{nM}$ or $1 \mu \mathrm{M})$ for 72 hours, analyzed by flow cytometry. Results are represented as mean \pm SD of 3 independent experiments. ${ }^{* *} P<0.01$ by 1 -way ANOVA with Bonferroni's post hoc test. (D) Colony formation assay in 4 sensitive (A2780, OVCAR5, ICROV1, and POVC1) and 4 resistant (OVCAR3, OVCAR4, SKOV3 and POVC20) OV commercial cell lines and patient-derived cells. Cells were treated with vehicle or trametinib (100 or $500 \mathrm{nM})$ for $10-12$ days. Images are representative of 3 independent experiments. (E) Immunoblot analysis of MEK/ERK signaling in sensitive and resistant cells treated with increasing concentrations of trametinib for 6 hours. (F) Immunoblot analysis of MEK/ERK signaling in sensitive and resistant cells treated with $100 \mathrm{nM}$ trametinib at indicated time points.

resistant and sensitive cells by performing chromatin immunoprecipitation-sequencing (ChIP-Seq) for H3K27ac, H3K4me1, and H3K4me3. Distributions of H3K27ac, H3K4me1, and H3K4me3 across the genome were similar between parental and resistant
A2780 cell lines (Figure 3A). Both H3K27ac and H3K4me3 peaks were enriched at transcription start sites (TSSs), where H3K4me1 was generally depleted (Figure 3B). We defined promoters as regions with $\mathrm{H} 3 \mathrm{~K} 27 \mathrm{ac}^{+} / \mathrm{H} 3 \mathrm{~K} 4 \mathrm{me}^{+}$peaks and within $\pm 2 \mathrm{~kb}$ of any 
A

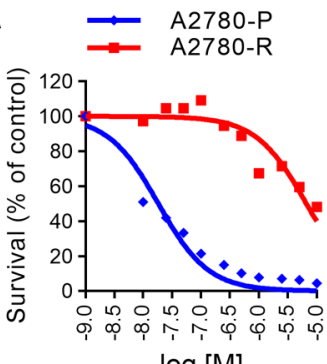

$\log [\mathrm{M}]$

A2780-P IC ${ }_{50}=18.3 \mathrm{nM}$

$\mathrm{A} 2780-\mathrm{R} I \mathrm{C}_{50}=6.63 \mu \mathrm{M}$

D

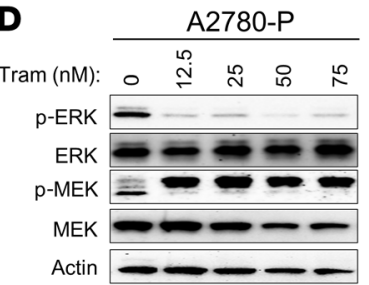

F

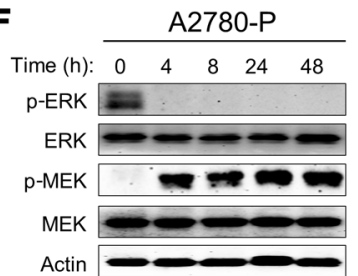

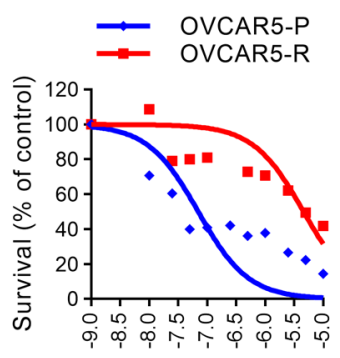

$\log [\mathrm{M}]$

OVCAR5-P IC ${ }_{50}=70.6 \mathrm{nM}$

OVCAR5-R IC ${ }_{50}=4.63 \mu \mathrm{M}$
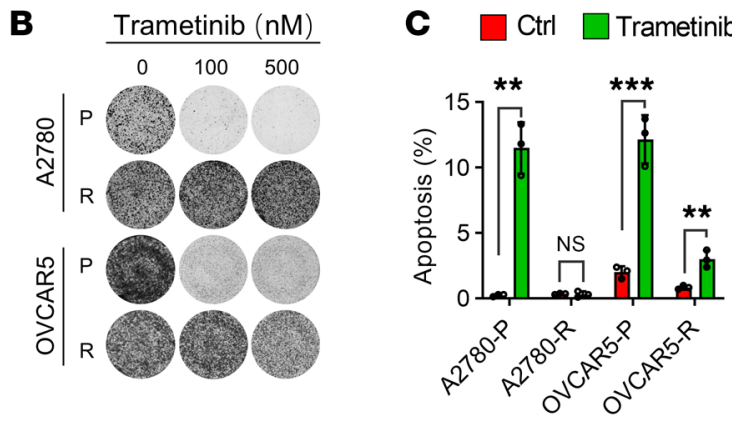

A2780-R
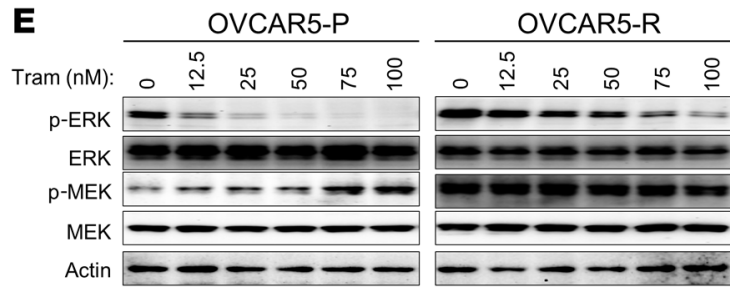

$\mathbf{G}$

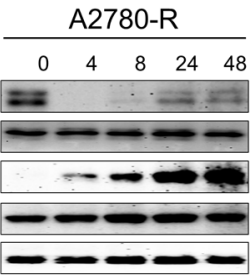

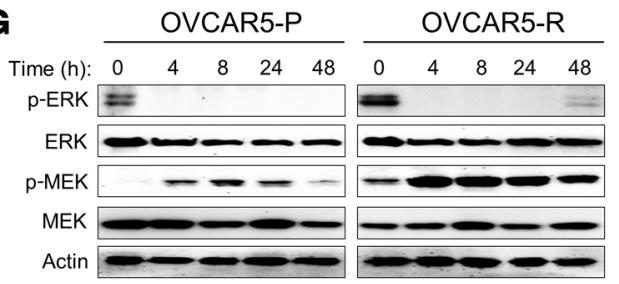

Figure 2. Persistent ERK activation is associated with acquired resistance to MEK inhibitor in OV. (A) A2780-P, A2780-R, OVCAR5-P, and OVCAR5-R cells were treated with the indicated concentrations of trametinib. The number of viable cells was measured at 96 hours. Data represent the mean of 3 biological replicates. IC $\mathrm{C}_{50}$ was calculated using GraphPad Prism software. (B) Colony formation assay of parental and resistant A2780 and OVCAR5 cells treated with vehicle or trametinib (100 or $500 \mathrm{nM}$ ). Images are representative of 3 independent experiments. (C) Quantification of the apoptotic cells among A2780-P/R and OVCAR5-P/R cells treated with vehicle or trametinib for 72 hours, analyzed by flow cytometry. Results are represented as mean \pm SD of 3 independent experiments. ${ }^{* *} P<0.01,{ }^{* *} P<0.001$ by unpaired Student's $t$ test. ( $\mathbf{D}$ and $\mathbf{E}$ ) Immunoblot analysis of MEK/ERK signaling in A2780 (D) and OVCAR5 (E) cells with their counterpart resistant lines treated with increasing concentrations of trametinib for 48 hours. (F and $\mathbf{G})$ Immunoblot analysis of MEK/ERK signaling in A2780 (F) and OVCAR5 (G) cells with their counterpart resistant lines treated with $50 \mathrm{nM}$ (A2780) or $100 \mathrm{nM}$ (OVCAR5) trametinib at indicated time points.

TSS in refTSS (version 3.1) and enhancers as regions outside $\pm 2 \mathrm{~kb}$ of all TSSs and having $\mathrm{H} 3 \mathrm{~K} 27 \mathrm{ac}^{+} / \mathrm{H} 3 \mathrm{~K} 4 \mathrm{me} 1^{+}$peaks. Promoters and enhancers are assigned to the nearest gene within $1000 \mathrm{~kb}$. The definition satisfied exactly specific criteria for this designation, which is consistent with previous studies (34-36).

Substantial enhancer and promoter reprogramming was observed in the A2780 resistant cell line. Merging 68,245 H3K27ac peaks in parental and 65,259 in resistant A2780 cells gave 87,146 peaks, 39,516 of which overlapped with TSS $\pm 2 \mathrm{~kb}$ regions while the remaining 47,630 did not. Of the $47,630 \mathrm{H} 3 \mathrm{~K} 27 \mathrm{ac}$ peaks located outside TSS $\pm 2 \mathrm{~kb}$ regions, 21,837 of them had overlapped H3K4me1 peaks, i.e., enhancer regions, of which 5544 (25.4\%) were common in both parental and resistant A2780 cell lines. There were 9979 (45.7\%) gained and 6314 (28.9\%) lost enhancers in the resistant A2780 cell line. Overlapping the 39,516 H3K27ac peak regions with $\mathrm{H} 3 \mathrm{~K} 4 \mathrm{me} 3$ peaks gave 26,053 promoter regions, with 17,980 (69.0\%) shared by parental and resistant A2780 cells. There were 2995 (11.5\%) lost and 5078 (19.5\%) gained promoters in the resistant A2780 cell line (Figure 3C). Gained enhancer and promoter regions in A2780 resistant cells showed elevated H3K27ac levels and a marked increase of H3K4me1. Similarly, at lost enhancers, both $\mathrm{H} 3 \mathrm{~K} 27 \mathrm{ac}$ and $\mathrm{H} 3 \mathrm{~K} 4 \mathrm{me} 1$ were reduced. Concordant changes of $\mathrm{H} 3 \mathrm{~K} 4 \mathrm{me} 3$ and $\mathrm{H} 3 \mathrm{~K} 27 \mathrm{ac}$ were also observed at gained or lost promoter regions (Figure 3, D and E). These data suggested that massive enhancer reprogramming accompanied the acquired resistance to trametinib in OV.

To further investigate whether the transcriptional change was related to chromatin modification, we performed RNA sequencing (RNA-Seq) in A2780-P and A2780-R cells. Integrative analysis of transcriptome and chromatin landscape indicated that H3K27ac change was generally correlated with the expression fold changes of H3K27ac target genes (Figure 3F). Loss of H3K27ac signal in enhancer regions was strongly associated with transcriptional downregulation in resistant cells and vice versa. Integrating histone modifications with transcriptome data, we identified 81 upregulated and 88 downregulated genes in resistant versus sensitive cells $\left(|\Delta \mathrm{H} 3 \mathrm{~K} 27 \mathrm{ac}| \geq 100\right.$ counts per million; gene expression $\mid \log _{2}$ fold change $\mid \geq 1$ and adjusted $P$ value $\leq 0.05$; Supplemental Figure $3 \mathrm{~A}$ ). In addition, gene set enrichment analysis in the Kyoto Encyclopedia of Genes and Genomes (KEGG) pathway identified significant suppression of MAPK signaling pathway in the resistant A2780 cell line (normalized enrichment score $=-1.69$, nominal $P$ value $=$ 
A

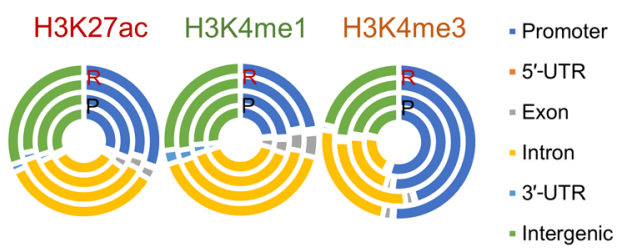

D

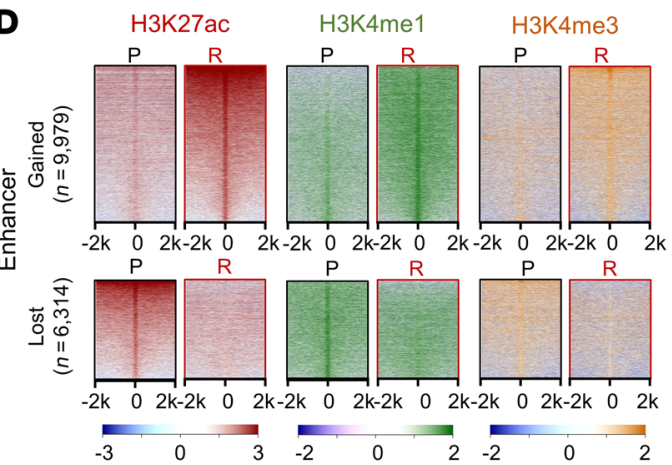

E

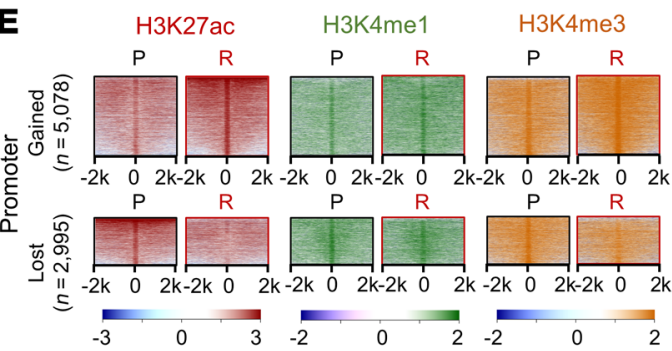

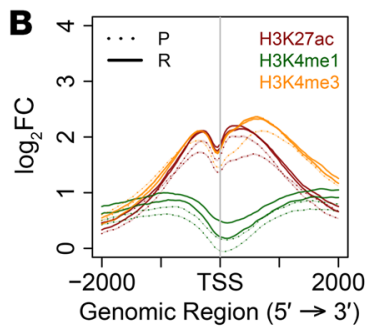

$\mathbf{F}$

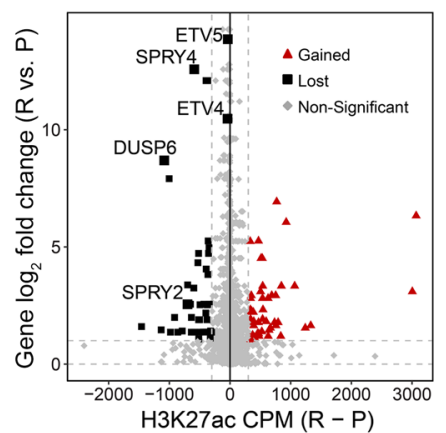

C

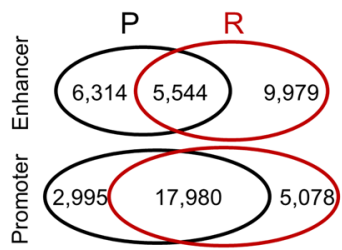

G

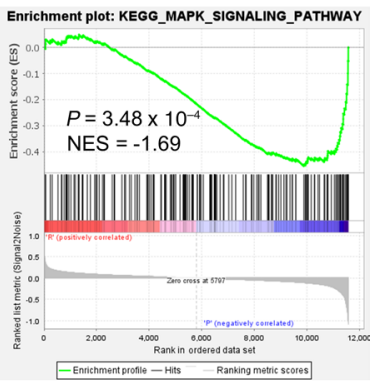

H

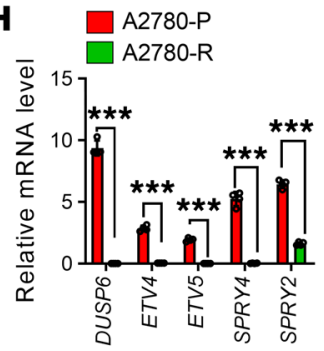

OVCAR5-P

OVCAR5-R

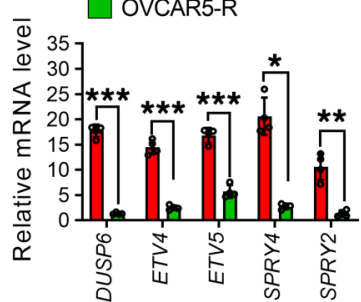

I chr12:89,703,103-89,754,791 chr17:41,603,214-41,625,708 chr5:141,688,000-141,772,673

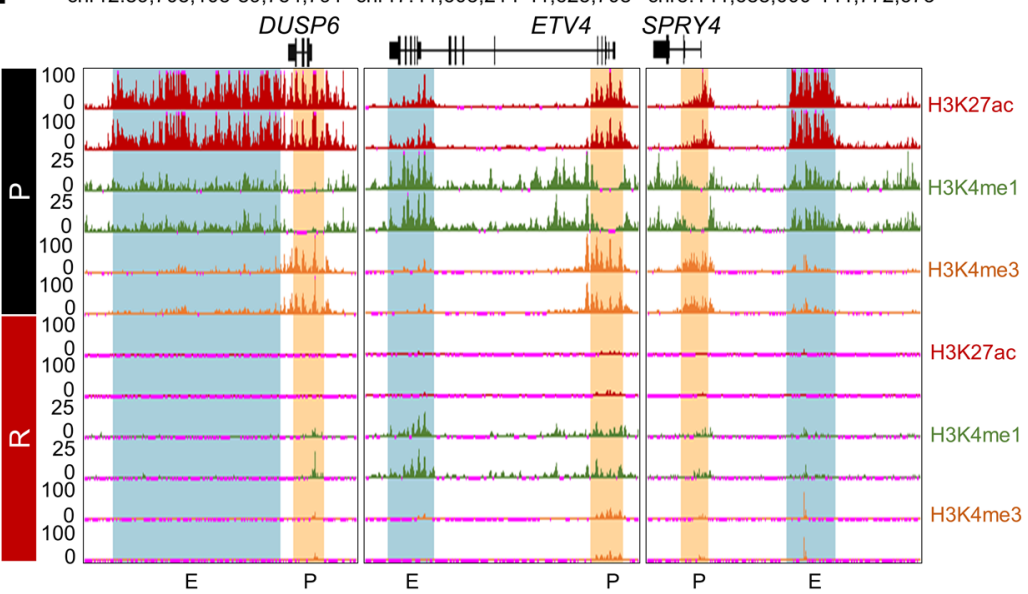

$J$

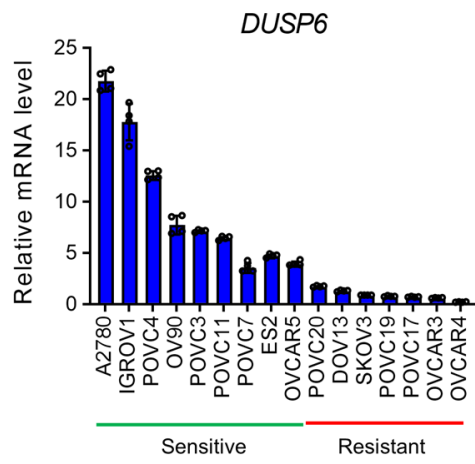

Figure 3. Enhancer reprogramming accompanies acquired resistance to MEK inhibitors. (A) Genomic distribution of H3K27ac, H3K4me1, and H3K4me3 peaks in A2780-P and A2780-R cells. (B) Plot of the average level of H3K27ac, H3K4me1, and H3K4me3 peaks centered at the TSS in A2780-P or A2780-R cells. (C) Venn diagram showing the number and overlaps of enhancers and promoters between A2780-P and A2780-R cells overlapped by MAnorm (https://github.com/shaolab/MAnorm). Common peaks merged. (D and E) Distribution of histone markers surrounding the summit of H3K27ac peaks, in gained or lost enhancer regions (D) and promoter regions (E). Black borders, A2780-P; dark red borders, A2780-R. Only one of the duplicates was plotted, as consistent results were observed in the other replicate. Each row represents 1 peak centered at the midpoint between two 2-kb flanking regions. (F) Scatterplot of the correlation between H3K27ac mark intensity and fold change of corresponding gene expressions. The horizontal axis illustrates the differences between resistant and parental cell lines, measured in counts per million mapped reads (CPM). Only genes of at least 4-fold change and H3K27ac changes of 500 CPM are shown. (C) Kyoto Encyclopedia of Genes and Genomes (KECG) pathway analysis enriched by downregulated genes in A2780-R (relative to A2780-P) showed that the MAPK pathway was significantly enriched. NES, normalized enrichment score. $(\mathbf{H})$ Relative mRNA levels of ERK transcriptional targets in A2780-P/R cells (left) and OVCAR5-P/R cells (right). Results are represented as mean \pm SD of 3 independent experiments. ${ }^{*} P<0.05$, ${ }^{* *} P<0.01$, ${ }^{* *} P<0.001$ by unpaired Student's $t$ test. (I) ChIP-Seq profiles show the ChIP-Seq signal ( $y$ axis, reads per million) for H3K27ac, H3K4me1, and H3K4me3 at genomic loci of DUSP6, ETV4, and SPRY4. (J) The results showed relative mRNA level of DUSP6 in 9 commercial cell lines and 7 patient-derived cells. Results are represented as mean \pm SD of 4 independent experiments. 
A

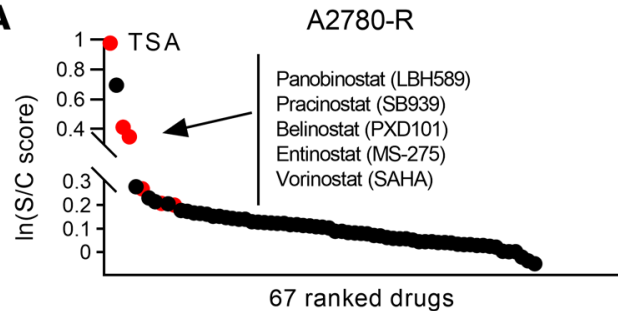

B

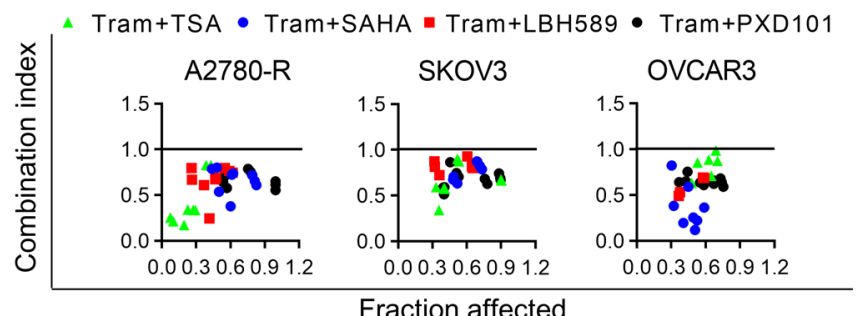

SKOV3

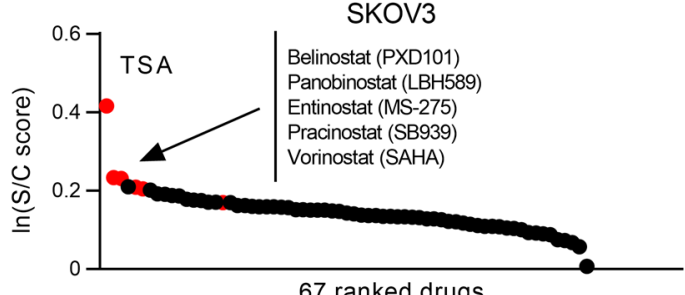

67 ranked drugs

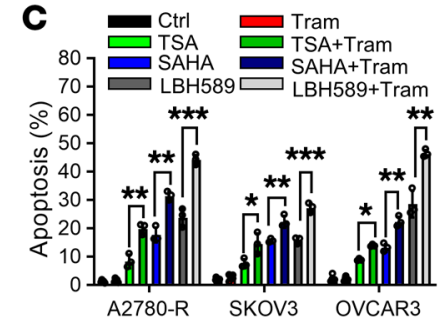

D

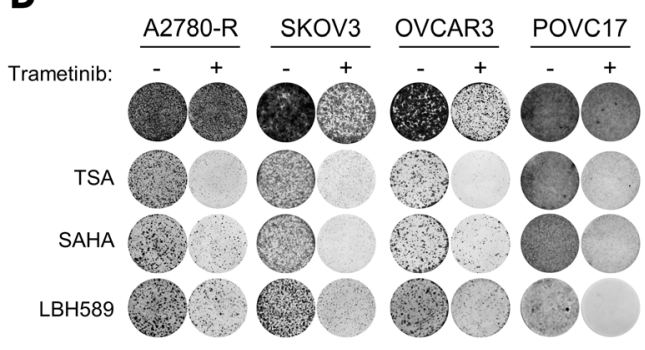

$\mathbf{F}$

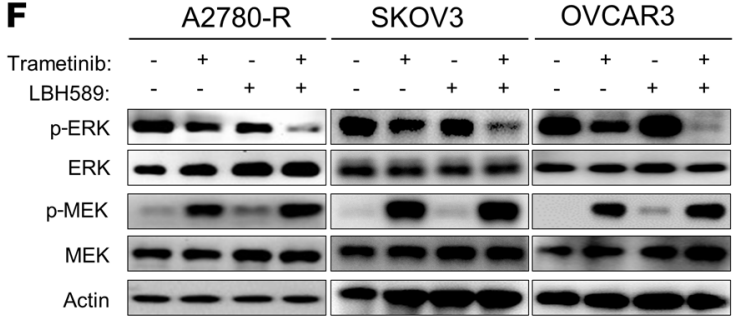

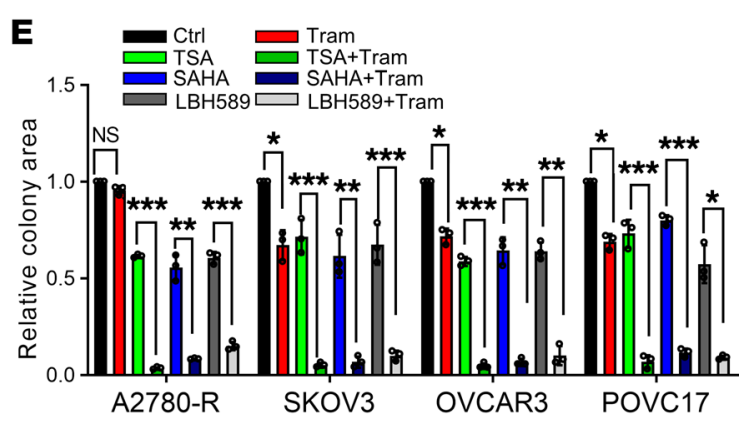

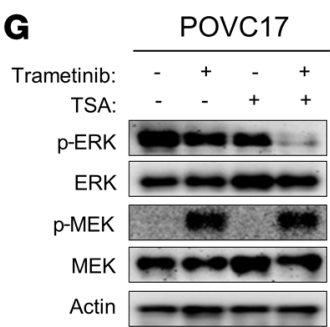

Figure 4. Combination drug screen identifies HDAC inhibitors as sensitizers to trametinib in resistant cells. (A) Graph showing the results of a drug screen performed in A2780-R and SKOV3 triplicates with 67 compounds either singly or in combination with $0.5 \mu \mathrm{M}$ trametinib. Data represent the mean of 3 biological replicates. Sixty-seven drugs on-screen are ranked according to the S/C (single/combination) score. Arrows highlight HDAC inhibitors (red). (B) Combination index of MEK and HDAC inhibitors in trametinib-resistant cell lines. Data represent the mean of 3 biological replicates. Combination index greater than 1 was defined as antagonism; combination index less than 1 was defined as synergy. (C) Apoptosis induced by combination of trametinib and $\mathrm{HDACi}$ in resistant cell lines. Cell lines were treated as indicated for 72 hours, after which annexin V/propidium iodide staining was performed, followed by flow cytometry. ( $\mathbf{D}$ and $\mathbf{E})$ Representative images (D) and quantification (E) of colony formation assay in resistant cells treated with vehicle, trametinib, HDACi, or their combination. (F) Immunoblot analysis of ERK and MEK activity in A2780-R, SKOV3, and OVCAR3 cells treated with vehicle, 100 nM trametinib, 25 nM LBH589, or their combination for 72 hours. (G) Immunoblot analysis of ERK and MEK activity in POVC17 cells treated with vehicle, $100 \mathrm{nM}$ trametinib, $50 \mathrm{nM}$ TSA, or their combination for 72 hours. (C and $\mathbf{E}$ ) Results are represented as mean $\pm \mathrm{SD}$ of 3 independent experiments. ${ }^{*} P<0.05$, ${ }^{* *} P<$ $0.01,{ }^{* *} P<0.001$ by 1 -way ANOVA with Bonferroni's post hoc test.

$3.48 \times 10^{-4}$; Figure $\left.3 \mathrm{G}\right)$. Interestingly, the top genes showing both downregulation and lost enhancer H3K27ac signals in resistant cells were those involved in the feedback inhibition of MEK/ERK signaling, including dual-specificity phosphatase 6 (DUSP6) (37, 38), the Ets variant transcription factors ETV4 and ETV5 (39-41), and the sprout homologs SPRY2 and SPRY4 $(42,43)$ (Figure 3F). This observation was further confirmed by real-time quantitative reverse transcriptase PCR (qRT-PCR) in both parental and resistant cells (Figure 3H). ChIP-Seq profiling for H3K27ac, H3K4me1, and H3K4me3 showed a loss in the enhancer/promoter activities for these genes (Figure 3I and Supplemental Figure 3B). Moreover, the expressions of these negative regulators of the MAPK pathway were suppressed not only in A2780-R and OVCAR5-R cells, but also in intrinsic-resistant cells compared with sensitive cells (Figure 3J and Supplemental Figure 3C). Altogether, these data suggested that the downregulation of negative regulators of the MAPK pathway by enhancer reprogramming could reactivate ERK activity, leading to trametinib resistance. 
A

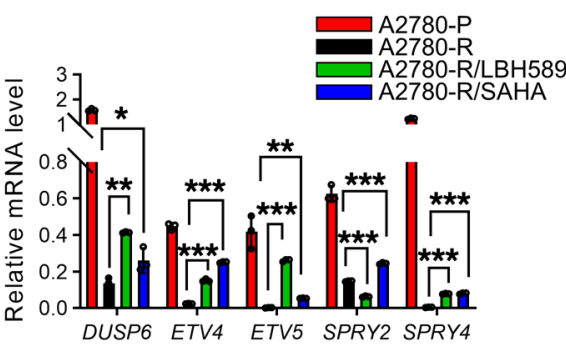

B

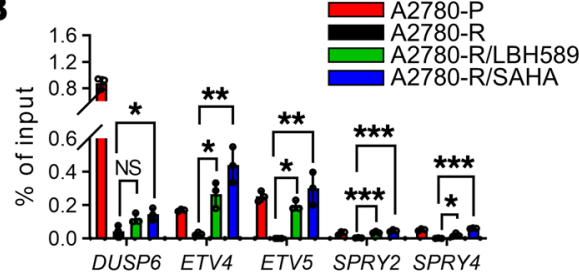

C

SKOV3

OVCAR3

POVC17
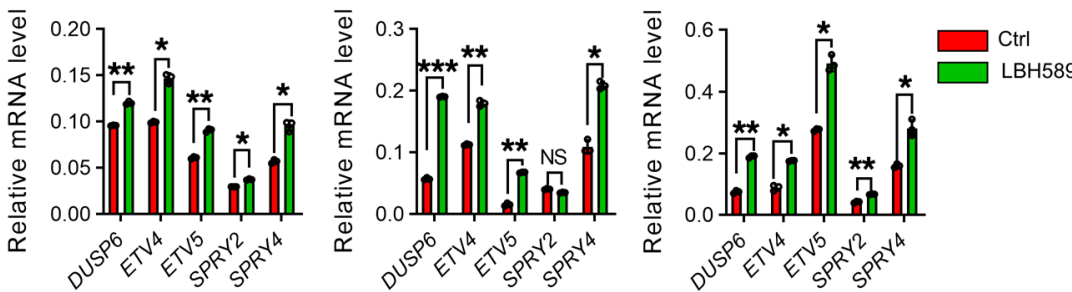

D SKOV3-H3K27ac

OVCAR3-H3K27ac
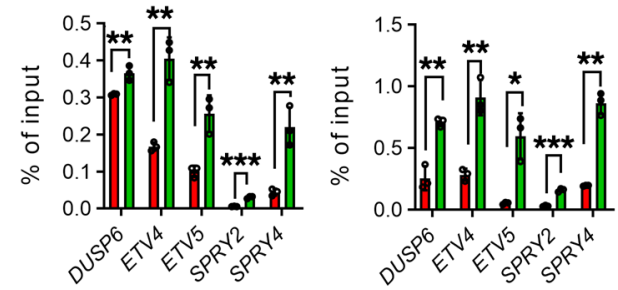

$\mathbf{F}$

E
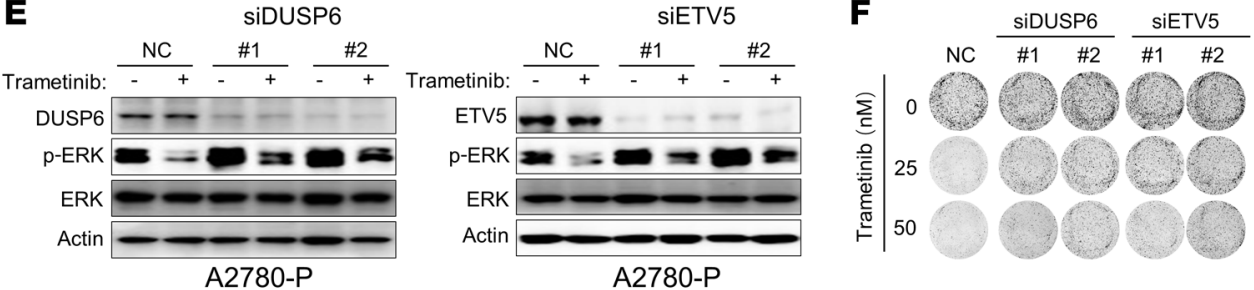

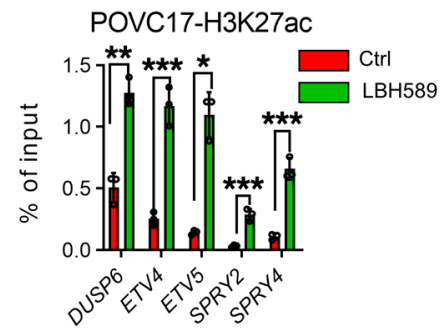

G
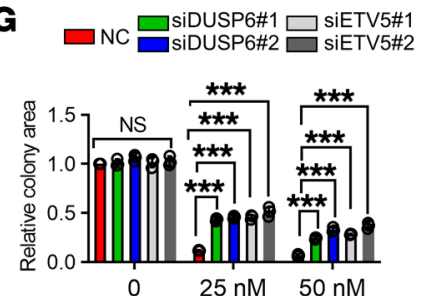

J

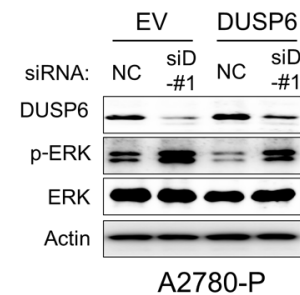

$\mathbf{N}$

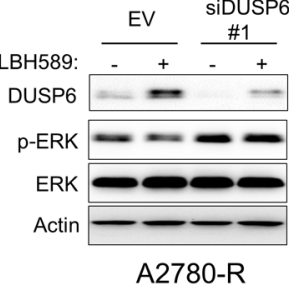

Figure 5. HDAC inhibitors reactivate repressive enhancers to block MAPK signaling restoration in trametinib-resistant cells. (A and $B$ ) $q R T-P C R$ (A) and ChIP-qPCR analysis of H3K27ac binding (B) of MAPK negative regulators in A2780-P and A2780-R cells treated with vehicle, SAHA, or LBH589. (C and D) qRT-PCR (C) and ChIP-qPCR analysis of H3K27ac binding (D) of MAPK negative regulators in resistant cells treated with vehicle or LBH589. (E) Immunoblot analysis of indicated proteins in A2780-P cells treated with trametinib after transfection with either negative control (NC), siDUSP6, or siETV5. (F and $\mathbf{G})$ Representative images (F) and quantification (G) of colony formation assay in A2780-P cells treated with trametinib after transfection with siRNA described in E. (H) Cell viability assay of the effect of DUSP6 re-expression in A2780-R or OVCAR5-R cells on trametinib sensitivity. (I) Immunoblot analysis of indicated proteins in A2780-R or OVCAR5-R cells with DUSP6 re-expression, treated with vehicle or trametinib. (J and $\mathbf{K})$ Immunoblot analysis of indicated proteins (J) and representative images (K) of colony formation assay in A2780-P cells treated with either siNC or siDUSP6 \#1 (targeting untranslated region) after stable expression of empty vector (EV) or DUSP6. (L and M) Representative images (L) and quantification (M) of colony formation assay in A2780-R cells treated with trametinib with or without HDACi after transfection with either siNC or siDUSP6. (N) Immunoblot analysis of indicated proteins in A2780-R cells treated with vehicle or LBH589 after transfection with siRNA described in $\mathbf{L}$. $(\mathbf{F}, \mathbf{K}$, and $\mathbf{L}$ ) Images are representative of $\mathbf{3}$ independent experiments. (A-D, $\mathbf{G}, \mathbf{H}$, and $\mathbf{M}$ ) Data are mean \pm SD of 3 independent experiments. (A, B, and $\mathbf{G}$ ) One-way ANOVA with Bonferroni's post hoc test; (C, D, and M) unpaired Student's $t$ test; (H) 2-way ANOVA with Tukey's post hoc test. ${ }^{*} P<0.05,{ }^{* *} P<0.01,{ }^{* *} P<0.001$. 
Combination drug screen identifies HDAC inhibitors as sensitizers to trametinib in resistant cells. To determine whether targeting of enhancer reprogramming is a potential therapeutic strategy to overcome resistance to trametinib, we performed a combination drug screen using 67 compounds that targeted epigenetic modifiers in both intrinsic-resistant SKOV3 cells and acquired-resistant A2780-R cells to evaluate the combined effect on cell viability with trametinib. The results showed that HDAC inhibitors were most enriched among those epigenetic drugs, showing a combinatorial effect with trametinib compared with single treatment (Figure 4A). To further determine the synergistic anticancer effect of trametinib in combination with HDAC inhibitors in trametinib-resistant cell lines, we performed combination index analyses, based on the Chou-Talalay combination index model, across 4 resistant cell lines and 1 patient-derived OV cells using HDAC inhibitors, including trichostatin A (TSA), suberoylanilide hydroxamic acid (SAHA), LBH589, and PXD101. We observed that all the combination index values were less than 1 , indicating a synergistic effect between HDAC inhibitors and trametinib (Figure 4B and Supplemental Figure 4A). These combinational effects were further evidenced by apoptosis and cell proliferation assays (Figure $4 \mathrm{C}$ and Supplemental Figure 4B), as well as colony formation assay (Figure 4, D and E). Having shown that HDAC inhibitors sensitized to the effects of trametinib, we then sought to determine whether HDAC inhibitors suppressed sustained reactivation of ERK activity in resistant cells to overcome the resistance to trametinib. We evaluated the MEK/ERK activities in cells treated with trametinib in the presence or absence of HDAC inhibitors. The results showed that reactivation of ERK was efficiently blocked by combination treatment in both intrinsic- and acquired-resistant cells (Figure 4, F and G and Supplemental Figure 4, C and D). Taken together, these findings demonstrated that HDAC inhibitors could overcome the resistance to trametinib by suppression of the ERK restoration in trametinib-resistant OV cells.

HDAC inhibitors reactivate repressive enhancers to block MAPK signaling restoration in trametinib-resistant cells. Having shown that HDAC inhibitors sensitized to the effects of trametinib and blocked restoration of MAPK signaling in both intrinsic- and acquired-resistant cells, we next examined whether HDAC inhibitors was able to induce the expression of the negative regulators of the MAPK pathway DUSP6, ETV4, ETV5, SPRY2, and $S P R Y 4$ through the modification of $\mathrm{H} 3 \mathrm{~K} 27 \mathrm{ac}$ levels at enhancers of these genes. qRT-PCR analysis in the acquired-resistant cell A2780-R (Figure 5A) and the intrinsic-resistant cells SKOV3, OVCAR3, and POVC17 showed that all of these genes were upregulated at the mRNA level by HDAC inhibitors except for SPRY2 in LBH589-treated A2780-R cells (Figure 5C and Supplemental Figure $5 \mathrm{~A})$. Interestingly, we found that there are 4 transcript variants encoding the same protein in the SPRY2 gene, which were differentially expressed in A2780-P and A2780-R cells as shown in the RNA-Seq data and validated by qPCR analysis (Supplemental Figure 5, B-D). The expression of transcript variant 1 (NCBI Reference Sequence NM_005842.3) was downregulated while other variants were upregulated and the total expression of SPRY2 was slightly increased in LBH589-treated A2780-R cells (Supplemental Figure 5, E and F). Immunoblot analysis further confirmed that the protein levels of these genes were upregulated by HDAC inhibitors (Supplemental Figure 5G), in agreement with previous findings that HDAC inhibitor monotreatment could inhibit p-ERK through upregulation of MAPK negative regulators (Figure 4). Consequently, combination treatment of HDAC/trametinib induced a synergistic blockade of p-ERK in resistant cells. Notably, we observed that LBH589 failed to significantly upregulate the expression of SPRY2 mRNA as compared with SAHA, while both HDAC inhibitors induced a dramatic increase in protein level in A2780-R cells, suggesting that LBH589 might have additional mechanisms of action that affect the expression of SPRY2, such as protein stability.

We observed that the amplitude of increase in mRNA level was small, but the $P$ value was significant and the protein levels of other MAPK negative regulators were dramatically increased by HDAC inhibitors, suggesting that the upregulation of these genes by HDAC inhibitors was real and consistent at both the protein and the mRNA level. Moreover, the MAPK pathway works as a signaling cascade, and any small changes induced by the HDAC inhibitors in any of the members of this pathway would likely have a profound amplified effect on the final targets, supporting our hypothesis that HDAC inhibitors may reactivate the MAPK negative regulators to reverse resistance to MEK inhibitor. Consistently, the H3K27ac levels at enhancers of these genes were enriched after treatment with HDAC inhibitor, supporting that the expression of these negative regulators was regulated by enhancer reprogramming in trametinib-resistant cells (Figure 5, B and D, and Supplemental Figure $5 \mathrm{H}$ ). To assure the specificity of the H3K27ac ChIP-qPCR signal, we included the known genomic regions enriched for H3K27ac represented by GAB2 and ACTB and lack of enrichment at known genomic regions represented by OUT and NC (Supplemental Figure 5, I and J) as positive and negative controls, respectively.

To further verify that the suppression of negative regulators of the MAPK pathway contributed to trametinib resistance, we performed siRNA-mediated knockdown of DUSP6, ETV5, or SPRY4 in A2780-P cells (Supplemental Figure 6A). The knockdown of DUSP6, ETV5, or SPRY4 could induce a rescue of p-ERK during trametinib treatment (Figure 5E and Supplemental Figure 6B) and impair the sensitivity to trametinib in colony formation assay (Figure 5, F and G, and Supplemental Figure 6, C and D). More importantly, ectopic expression of DUSP6 or SPRY4 could partially confer sensitivity to trametinib and attenuate sustained ERK activities in resistant cells (Figure 5, H and I, and Supplemental Figure 6, E and F). These findings are consistent with previous studies showing that restoration of DUSP6 or other MAPK negative regulators could resensitize the resistance to targeted therapy (44-48). In addition, we performed a rescue experiment and found that DUSP6 knockdown-induced resistance and ERK restoration were reversed by overexpression of DUSP6 in A2780-P cells (Figure 5, J and K, and Supplemental Figure $6 \mathrm{G})$. To further determine whether reactivation of DUSP6 mediates the effect of HDAC inhibitors on the trametinib response, we conducted siRNA-mediated DUSP6 knockdown in resistant cells to evaluate the combination effects of MEK/HDAC inhibitor. The results showed that DUSP6 knockdown significantly impaired the combination effect and HDAC inhibitor-induced DUSP6 was suppressed by DUSP6 knockdown, resulting in ERK 
A

SKOV3

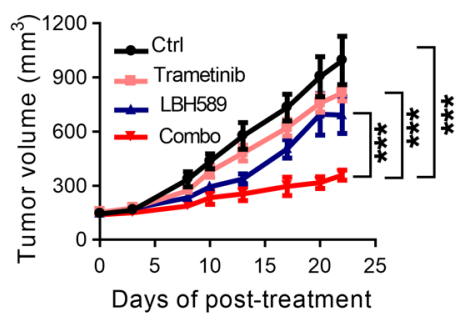

D

Ki67

p-ERK

Ki67

$\mathbf{F}$

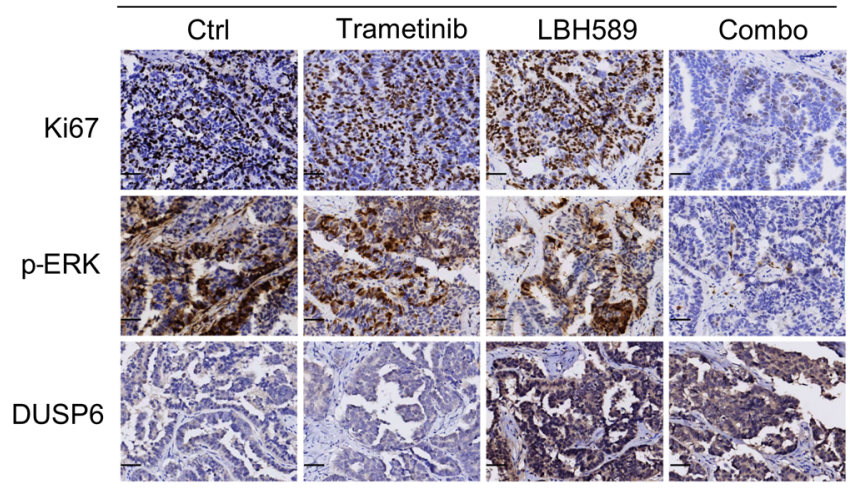

H

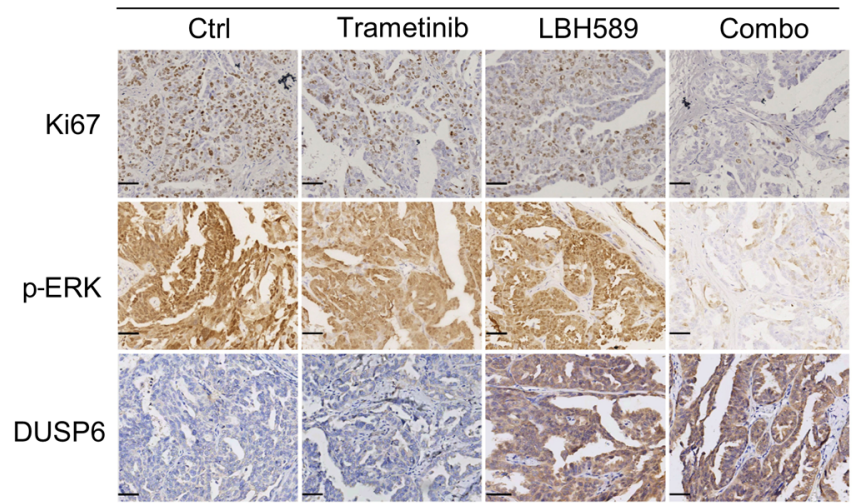

B

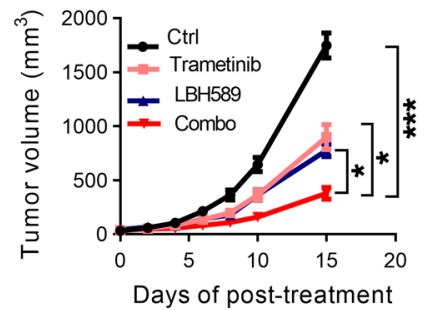

C

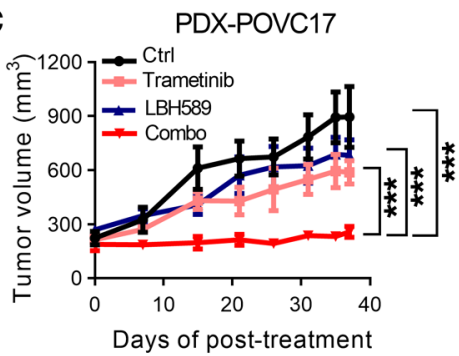

SKOV3

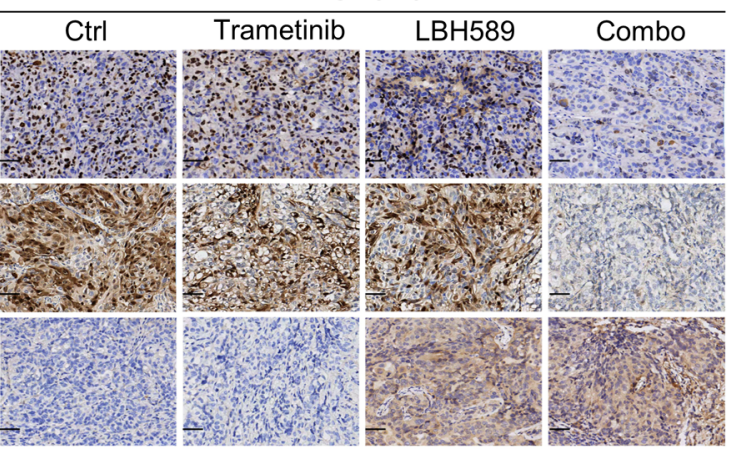

PDX-POVC15

G
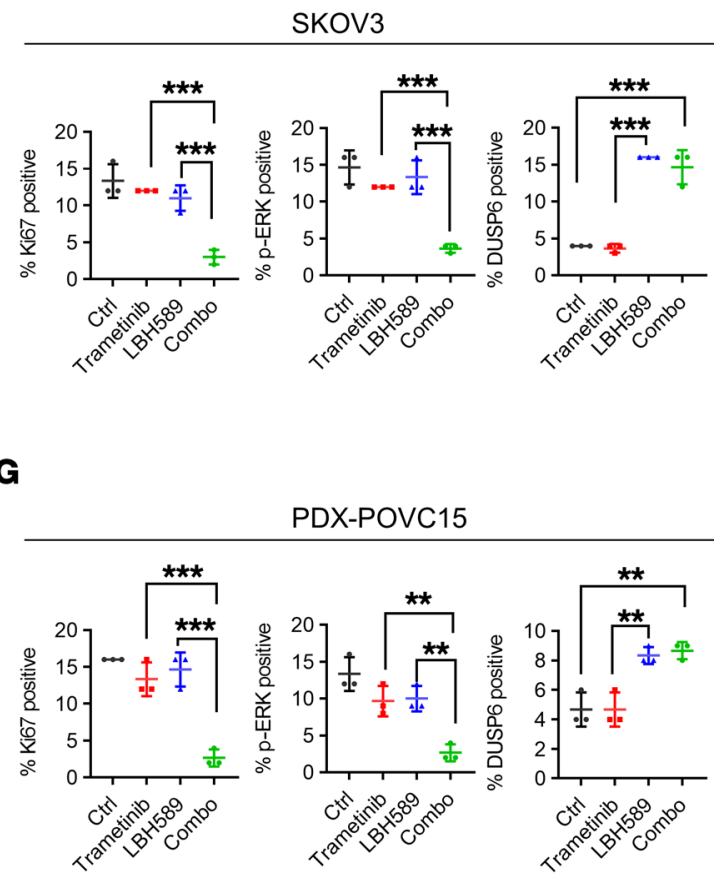

E

I

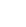


reactivation (Figure 5, L-N). Thus, these findings suggested that HDAC inhibitors could reactivate the expression of MAPK negative regulators through enhancer reprogramming and that changes in the expression of these genes in resistant cells played functional roles in mediating resistance.

HDAC inhibitor sensitizes resistance to MEK inhibitor in vivo. To investigate the effect of the combination of trametinib and HDAC inhibitor in vivo, we examined the efficacy and toxicity of this potential therapeutic combination in xenograft tumor models. In the SKOV3 xenograft model, LBH589 in combination with trametinib retarded tumor growth compared with LBH589 or trametinib alone (Figure 6A). In addition, LBH589, trametinib, or their combination was well tolerated, as demonstrated by the maintenance of body weight in the treatment group (Supplemental Figure 7A). Moreover, in 2 OV patient-derived xenograft (PDX) models (PDX-POVC15 and PDX-POVC17), whose primary cells were resistant to trametinib in vitro, combination treatment significantly inhibited tumor growth in both PDX models with modest side effects (Figure 6, B and C, and Supplemental Figure 7, B and C). Notably, we performed IHC staining for p-ERK and Ki67 as well as DUSP6 in all xenograft/PDX tumor samples. The IHC data showed that the combination treatment exerted dramatic suppression of p-ERK and inhibition of proliferation. In addition, either HDAC inhibitor or the combination significantly induced transcriptional reactivation of DUSP6 (Figure 6, D-I), which was consistent with findings in vitro. Together, these data support the notion that HDAC inhibitors could reactivate DUSP6 and resensitize the resistance to trametinib in vivo.

\section{Discussion}

In this study, we investigated the potential efficacy of, and mechanisms of resistance to, the MEK inhibitor trametinib in advanced OV using patient-derived cells and xenograft models. Our study identified enhancer reprogramming as a causative factor that mediated resistance to trametinib and further demonstrated the effectiveness of a drug combination strategy in inhibiting HDAC to overcome this resistance. Moreover, we identified potential predictive biomarkers, such as DUSP6, ETV4, and SPRY4, which were correlated with sensitivity to trametinib in OV.

The MAPK signaling pathway is frequently activated in advanced OV. Genetic alterations, including oncogenic activation of KRAS/BRAF and inactivation of NF1, have been associated with hyperactivation of the MAPK pathway in OV $(6,8)$, which can be measured by phosphorylated forms of key nodes of the pathway including p-ERK and p-MEK. Our findings confirmed the constitutive activation of the MAPK pathway in patient-derived OV cells and commercial cell lines as evidenced by the detection of p-ERK and p-MEK, indicating aberrant MAPK pathway as a potential target in advanced OV. MEK inhibitors, which impair the activation of MAP kinases and also block the aberrant activation of upstream RAS, have demonstrated promising results for cancer therapy (7). In line with a recent case report of a patient with heavily pretreated OV responding to trametinib (17), we showed that trametinib could indeed exert a profound therapeutic effect on OV by suppressing the MAPK signaling. However, chronic exposure to trametinib could lead to the acquisition of resistance and reactivation of $\mathrm{p}$-ERK, mirroring clinical observa- tions of eventual disease progression after an initial response to several months of treatment. In this study, we showed that trametinib response is not correlated with genetic alterations such as mutations of members of the MAPK pathways in both acquiredand intrinsic-resistant cells, indicating that epigenetic alterations are responsible for MEK inhibitor resistance.

A reversible transition of epigenetic states that causes dynamic chromatin modifications can lead to drug resistance $(49,50)$. For instance, acute reprogramming of enhancers has been observed in MEK inhibitor-treated triple-negative breast cancer models, and pharmacological inhibition of BRD4 can prevent the emergence of drug resistance (51). Consistent with these studies, we found that enhancer reprogramming could mediate resistance to trametinib. This observation indicated that targeting epigenetic pathways could be a promising strategy for overcoming acquired resistance to kinase inhibitors caused by enhancer reprogramming. Our integrated analysis using ChIP-seq and RNA-seq revealed that extensive enhancer reprogramming was accompanied with the suppression of MAPK negative regulators, such as DUSP6, SPRY4, and ETV4, upon acquisition of resistance to trametinib.. This was consistent with previous studies, in which the suppression of these MAPK negative regulators was associated with sensitivity to MEK inhibitor in various human cancers (52). In addition, genes involved in the feedback inhibition of MAPK signaling, such as DUSP6, $S P R Y 2$, and SPRY4, have been previously found to confer resistance to FGFR inhibition (53) in gastrointestinal stromal tumors and MET inhibition (54) in gastric cancer, suggesting that these MAPK negative regulators are key mediators of resistance to targeted therapy. Furthermore, DUSP6 knockdown contributed to resistance to targeted therapies including BRAF inhibition in melanoma and FGFR inhibitors in lung cancer $(45,55)$, whereas DUSP6 restoration increased sensitivity to ALK inhibitors (44). We also demonstrated that depletion of these MAPK negative regulators could confer resistance to trametinib in sensitive cells, whereas overexpression of those genes could impair resistance to trametinib in acquired-resistant cells. Together, these findings suggested that relief of feedback inhibition of MAPK signaling could attenuate sensitivity to trametinib in OV.

Recent studies indicated that HDAC inhibitors could enhance the antitumor activity of MEK inhibitors in various cancers, including lung cancer, uveal melanoma, and melanoma (56-59). The HDAC inhibitor belinostat activated FOXOs and upregulated expression of BIM and p21 to increase sensitivity to trametinib in non-small cell lung carcinoma with KRAS mutation (56). Increased ZEB1 and low IL17RD expression were reported to be associated with therapeutic resistance to MEK inhibition in $K R A S$-mutant lung tumors. Suppression of ZEB1 by the HDAC inhibitor mocetinostat sensitized resistant cancer cells to MEK inhibition (57). In melanoma, AR42, a class I/II HDAC inhibitor, potentiated cell toxicity of the multi-kinase inhibitor pazopan$\mathrm{ib}$ in trametinib/dabrafenib-resistant melanoma cells partially through inducing autophagy (58). In addition, GPCR-mediated YAP activation and RTK-driven AKT signaling were reported to contribute to MEK inhibitor resistance in uveal melanoma cells. HDAC inhibitor suppressed the adaptive AKT and YAP signaling to sensitize to MEK inhibition (59). These findings suggested 


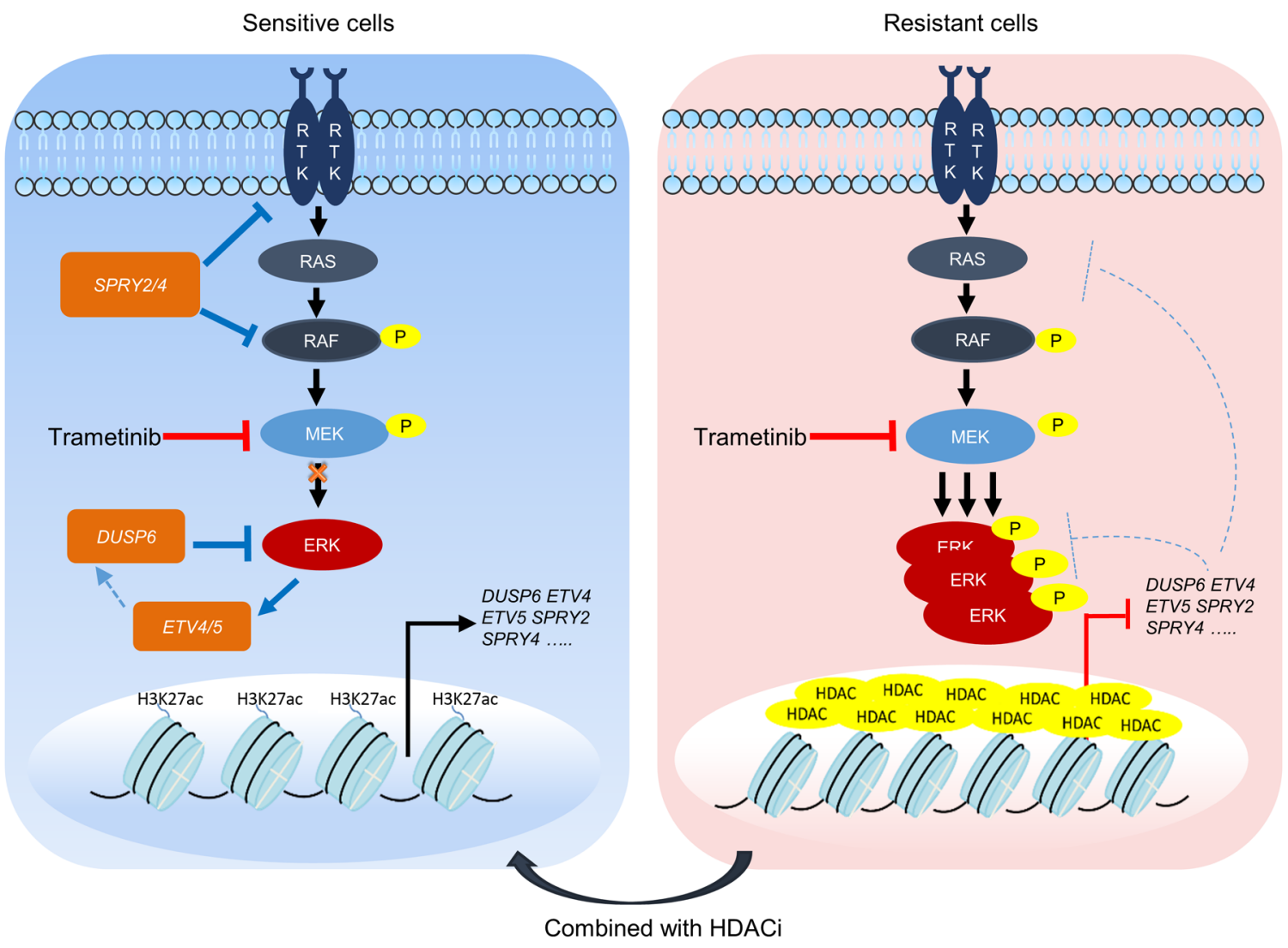

Figure 7. Schematic model illustrating the role of enhancer reprogramming in modulating sensitivity to MEK inhibitors in OV. In sensitive cells (left), the negative regulators of the MAPK pathway, including SPRY2/4, DUSP6, and ETV4/5, are expressed with active enhancers. Trametinib constitutively inhibits ERK activity. In resistant cells (right), MAPK negative regulators are suppressed as a result of enhancer reprogramming from the active enhancer to the repressive enhancer, leading to relief of feedback inhibition of MAPK signaling and conferring trametinib resistance. HDAC inhibitors can attenuate the enhancer reprogramming and upregulate the negative regulators of the MAPK pathway, resulting in a more complete suppression of ERK activity and overcoming the resistance to trametinib.

that HDAC inhibition could sensitize to MEK inhibition through distinct underlying mechanisms in different cancer types. In our study, we demonstrated that enhancer reprogramming, which caused the suppression of the MAPK negative regulators, rather than genetic alterations in certain genes is a causative factor that mediated resistance to trametinib (Figure 7). Consequently, HDAC inhibitors were able to reverse the chromatin structure of resistant cells to a sensitive state and eventually increase the expression of these MAPK negative regulators, leading to p-ERK suppression and overcoming MEK inhibitor resistance (Figure 7). Our findings not only unveiled potential resistance mechanisms that can be exploited for MEK-targeted therapy to overcome drug resistance in OV but also uncovered potential predictive biomarkers that could guide therapeutic strategies.

In conclusion, we found that resistance to MEK inhibitor was associated with enhancer reprogramming leading to a refractory ERK signaling. The addition of HDAC inhibitors could overcome the resistance to trametinib through epigenetic reprogramming. Overall, our findings highlight the importance of considering epigenetic drugs to overcome the acquisition of drug resistance in targeted therapy. Further clinical investigation that combines MEK and HDAC inhibitors in advanced OV, and exploration of this combination in other solid tumors, should be considered in the future.

\section{Methods}

Cell lines and reagents. All commercial cell lines were purchased from ATCC, except for A2780, COV362, and COV504 (obtained from the European Collection of Authenticated Cell Cultures), KURAMOCHI (purchased from the Japanese Collection of Research Bioresources Cell Bank), and DOV13 (obtained from BioVector NTCC). No authentication of cell lines was done by the authors. The cells were cultured in RPMI 1640 plus 10\% FBS, except for CAOV3 (DMEM plus 10\% FBS) and ES2 (McCoy's 5A plus 10\% FBS). All cell lines were maintained at $37^{\circ} \mathrm{C}$ in a humidified chamber in the presence of $5 \% \mathrm{CO}_{2}$ and were confirmed to be free of mycoplasma contamination every month. Cells used for experiments were between 3 and 20 passages from thawing. Trametinib, panobinostat (LBH589), and vorinostat (SAHA) were purchased from Selleck Chemicals. TSA was purchased from Cell Signaling Technology. Epigenetic drug library was purchased from Selleck Chemicals. Stock solutions were diluted in DMSO and stored according to the manufacturer's protocols.

Patient-derived xenograft model and primary cell culture. Patient tumor tissues were collected from female patients undergoing surgery at the Sun Yat-sen University Cancer Center. Informed consent was obtained from the patients who provided the tissue, and all procedures were approved by the medical ethics committee of the Sun Yat-sen University Cancer Center. Tumor tissue was examined by a 
pathologist to determine the tumor type and grade. Detailed patient information is shown in Supplemental Table 1. Tumor fragments were rinsed in saline and cut into small pieces for either patient-derived cells or xenografts. For the patient-derived xenograft (PDX) model, cut tumor tissue was implanted in the right or left axilla of NOD/SCID mice. After the tumor volumes reached approximately $1000 \mathrm{~mm}^{3}$, the mice were sacrificed. Tumor masses were isolated and subsequently passaged to new NOD/SCID mice for next generation. The third-generation tumor that had a stable growth rate was considered as a successful PDX model. We routinely compared the histopathological features of the passaged tumors with those of the original samples to confirm that the passaged tumors retained the morphological features of their original counterparts. About half of the patient sample was for the PDX models, while the other half was for the POVC cell lines. For patient-derived cells, the bulk tumors were treated with a tumor dissociation kit (MACS, Miltenyi Biotec) according to the manufacturer's protocols. Cells filtered by a $70 \mu \mathrm{m}$ strainer were cultured using DMEM/F12 (1:1) basic (Gibco) supplemented with $10 \%$ FBS, $10 \mathrm{ng} / \mathrm{mL}$ EGF (PeproTech), $10 \mathrm{mg} / \mathrm{mL}$ insulin, $0.5 \mu \mathrm{g} / \mathrm{mL}$ hydrocortisone, $10^{-10} \mathrm{M}$ cholera toxin, $2 \times 10^{-9} \mathrm{M}$ triiodo-L-thyronine, and $1.8 \times 10^{-4} \mathrm{M}$ adenine and containing penicillin and streptomycin (Gibco).

Establishment of cell lines with acquired resistance to trametinib. A2780 and OVCAR5 cells were grown with increasing concentrations of trametinib. Subconfluent cells were treated with trametinib at $\mathrm{IC}_{50}$ dose (both $25 \mathrm{nM}$, based on 96-hour cytotoxicity assays) for two 3-day periods, and surviving cells were maintained for another 4 days. The process was repeated once, and the remaining cells were cultured in the presence of drug for 3 additional passages. Resistant cells were maintained in the presence of trametinib, and the same passaged parental cells were used in various cellular assays.

Cell viability and colony formation assay. Two thousand cells were seeded in a 96-well plate for 24 hours and treated with indicated drugs for 96 hours. The number of viable cells was measured by the CellTiter-Glo Luminescent Cell Viability Assay (Promega). Luminescent signals were detected by a Tecan Infinite M200 Pro plate reader. All conditions were tested in triplicate, unless otherwise noted. Drug curves and $\mathrm{IC}_{50}$ values were generated using GraphPad Prism 8 (GraphPad Software). For colony formation assay, $1 \times 10^{4}$ cells were seeded in a 6-well plate and treated with indicated drugs for 10-12 days. The fresh medium was replaced every 3 days. Surviving colonies were fixed with methanol for 5 minutes and stained with crystal violet. They were quantified using ImageJ/Fiji software (https://fiji.sc/).

Apoptosis assay. A total of $1 \times 10^{5}$ cells were seeded in a 6-well plate and treated with indicated agents for 72 hours. Apoptotic cells were quantified using the Annexin V-FITC Apoptosis Detection Kit (Vazyme) according to the manufacturer's protocol. All experiments were performed in triplicate. Data were acquired and analyzed using BD LSRFortessa X-20 (BD Biosciences) and Spectral Cell Analyzer SP6800Z (Sony).

Combinatorial drug screen. A2780-R and SKOV3 cells were subjected to a combinatorial drug screen with a customized epigenetics compound library (Selleck Chemicals; Supplemental Table 2). Two thousand cells were seeded into a 96-well plate and treated with 67 compounds in the drug screen in the absence or presence of trametinib for 96 hours. Cell viability was assessed using CellTiter-Glo reagent (Promega) according to the manufacturer's instructions. Differential drug sensitivity was determined by the $\mathrm{S} / \mathrm{C}$ score, which is defined as DMSO-normalized viability of epigenetic library drug (single) divided by DMSO-normalized viability of trametinib + epigenetic library drug (combination).

Combination index analysis. Trametinib-resistant cells were simultaneously exposed to indicated concentrations of trametinib and HDAC inhibitors for 96 hours, and cell viability was subsequently assessed using CellTiter-Glo reagent (Promega). Combination index values were determined by the inhibition rate of the cells and calculated using CalcuSyn software (http://www.biosoft.com/w/calcusyn.htm). Combination index values less than 1 indicated a synergistic interaction between compounds, while those equal to or more than 1.0 indicated additivity or antagonism.

Immunoblotting. The detailed procedure of immunoblotting was described in a previous publication (60). Briefly, cell pellets were lysed using RIPA buffer (Roche). Protein concentrations were determined using a Bradford assay (Bio-Rad). An equal amount of protein was subjected to SDS-PAGE and subsequently transferred to the PVDF membrane (Bio-Rad). After blocking in 5\% BSA (Sigma-Aldrich) and incubation with appropriate primary antibodies and secondary antibodies, immunoblotting was developed with ECL Western Blotting Detection Reagents (GE Healthcare Life Sciences) and detected with a Bio-Rad ChemiDoc MP imaging system. The primary antibodies used were as follows: ERK1/2 (1:2000; 4696S), p-ERK1/2 (1:2000; 4370S), MEK (1:1000; 9126S), p-MEK (1:1000; 9154S), $\beta$-actin (1:2000; 8456S), and SPRY2 $(1: 1000 ; 14954 \mathrm{~S})$ were purchased from Cell Signaling Technology; DUSP6 (1:1000, pa5-15552; or 1:1000, ab76310) was obtained from Invitrogen or Abcam and ETV5 (1:1000; ab102010) was from Abcam; and SPRY4 (1:2000; 22765-1-AP) was from Proteintech. The secondary antibodies were HRP-conjugated anti-rabbit and anti-mouse (GE Healthcare Life Sciences NA934 and NA931).

RNA-Seq and data analysis. A2780-P and A2780-R were harvested after 24 hours of seeding, and total RNA was isolated using the RNeasy Mini Kit (Qiagen). RNA sequencing libraries were prepared using TruSeq Stranded RNA HT kit (96 samples) Ribo-Zero Gold (Illumina) according to the manufacturer's protocol and were sequenced on a Novaseq $\mathrm{S} 6000$ sequencing system (Illumina). Library integrity was assessed on an Agilent 2100 Bioanalyzer.

Quality-control passed reads were gained using fastp software (version 0.12.5; ref. 61) and were subsequently mapped to the human reference genome (GRCh37, hg19) using STAR (62) with GENCODE annotation (gencode.v19.annotation.gtf) and recommended settings from the STAR manual. Gene expression levels were quantified with RSEM (version 1.3.3; ref. 63). Differentially expressed genes were identified with DESeq2 (version 1.28.1; $\mid \log _{2}$ fold change $\mid \geq 1$ and adjusted $P$ value $\leq 0.05$; ref. 64). Gene set enrichment analysis (GSEA) was performed using GSEA (version 4.0.4; ref. 65). We used customized R scripts to generate visualizations.

qRT-PCR. Total RNA was isolated using the RNeasy Mini Kit (Qiagen); cDNA was subsequently prepared using TranScript All-in-One First-Strand cDNA Synthesis SuperMix for RT-PCR (One-Step gDNA Removal). qRT-PCR was performed following the instructions of PerfectStart Green qPCR SuperMix (TransGen Biotech). 18S was used as an endogenous housekeeping gene for normalization. The primer pairs of the genes used for quantitative qRT-PCR are shown in Supplemental Table 3. The mRNA levels of these genes were determined as the mean of the Ct values obtained from the couple of primers. Data are shown as relative gene expression. 
ChIP and ChIP-qPCR. Cells were treated with HDAC inhibitors as indicated, cross-linked for 10 minutes at room temperature by $1 \%$ formaldehyde, and quenched with $0.125 \mathrm{M}$ glycine for 5 minute. Subsequently, the cross-linked cells were washed with TBSE buffer (20 $\mathrm{mM}$ Tris- $\mathrm{HCl} \mathrm{pH} 7.5,1 \mathrm{mM}$ EDTA, $150 \mathrm{mM} \mathrm{NaCl}$ ) and stored at $-80^{\circ} \mathrm{C}$. The frozen cross-linked cells were lysed (50 mM Tris- $\mathrm{HCl}, \mathrm{pH}$ 8.0; 10 mM EDTA; 1\% SDS) and sonicated on ice using Bioruptor (Diagenode) for 16 cycles (on and off session at 30 seconds each). Sonicated lysates were precleared with protein $\mathrm{G}$ Dynabeads (Invitrogen) for 1 hour at $4^{\circ} \mathrm{C}$ and were incubated overnight at $4^{\circ} \mathrm{C}$ with magnetic beads bound with antibodies against H3K27ac (Abcam ab4729), H3K4me1 (EMD Millipore 07-436), and H3K4me3 (EMD Millipore 07-473) and normal rabbit IgG (EMD Millipore 12-370). The immunoprecipitations were washed 4 times and were eluted in elution buffer $(50 \mathrm{mM}$ Tris-HCl, pH 8.0; 10 mM EDTA; 1\% SDS) for the subsequent reversed cross-linked process by incubation with pronase at $42^{\circ} \mathrm{C}$ for 2 hours and $68^{\circ} \mathrm{C}$ for 6 hours. The reversed cross-linked DNA was purified by Phenol-Chloroform-Isoamyl Alcohol (Thermo Fisher Scientific) and ethanol precipitation. ChIP-qPCR assay was performed using SYBR Green PCR Master Mix (Applied Biosystems). The primer sequences are listed in Supplemental Table 3. The enrichment of specific genomic regions was calculated relative to the input DNA followed by normalization to the respective control IgG values.

ChIP-Seq. ChIP DNA and input DNA were amplified using the GenomePlex Single Cell Whole Genome Amplification Kit (Sigma-Aldrich). After digestion with BpmI (New England Biolabs), ChIP DNA (30 ng) was used for library construction using NEBNext ChIP-Seq Library Prep Master Mix Set for Illumina (New England Biolabs) according to the manufacturer's recommended protocol. Libraries were sequenced using an Illumina HiSeq 2500.

ChIP-Seq data analysis. For preprocessing, the quality of next-generation raw data was verified using FastQC (version 0.11.8; ref. 66). ChIP-Seq data were trimmed by $10 \mathrm{bp}$ from both ends to remove adapter sequences using Trim Galore (version 0.6.3, https://www. bioinformatics.babraham.ac.uk/projects/trim_galore/). Trimmed reads were mapped to human reference genome hg19 (hs37d5) using BWA-MEM (version 0.7.17-r1188; ref. 67). Mappings with mapping qualities above 10 were retained and sorted using SAMtools (version 1.9; ref. 68), after which duplicates were removed using the Picard toolkit (MarkDuplicates version 2.20.3, http://broadinstitute.github. io/picard/).

Peaks of H3K27ac (narrow), H3K4me1 (broad), and H3K4me3 (narrow) were called using MACS2 (version 2.1.2; settings: -f BAMPE -q 0.01 -g hs -B --trackline --SPMR; refs. 69, 70). Regions of peaks within ENCODE Blacklist regions were excluded (71). Only common peaks of at least 100 bp shared by the duplicated samples were retained for further analysis. Peaks were annotated with ChIPseeker (version 1.20.0; ref. 72) and visualized using ngs.plot.r (version 2.61; ref. 73). Promoters were regions with $\mathrm{H} 3 \mathrm{~K} 27 \mathrm{ac}^{+} / \mathrm{H} 3 \mathrm{~K} 4 \mathrm{me} 3^{+}$peaks and within $\pm 2 \mathrm{~kb}$ of any transcription start site (TSS) in refTSS (version 3.1; ref. 74). Enhancers were regions outside $\pm 2 \mathrm{~kb}$ of all TSSs and with $\mathrm{H} 3 \mathrm{~K} 27 \mathrm{ac}^{+} / \mathrm{H} 3 \mathrm{~K} 4 \mathrm{me}^{+}$ peaks. BEDOPS (75) and BEDTools (76) were used to process the BED files. GREAT (77) was used to identify the targeted genes of promoters and enhancers (single nearest gene within $1000 \mathrm{~kb}$ ).

To quantify changes in histone marks, recurrent peaks in duplicates of parental and resistant cell lines were merged, forming a list of promoter/enhancer candidate regions. Reads within promoter/ enhancer candidates were quantified using HTSeq-count (version 0.11.2; ref. 78). Read counts are normalized to counts per million (CPM) for comparisons. DeepTools bamCoverage generated the bigWig files for visualization in Integrative Genomics Viewer (version 2.4.19; ref. 79). Customized R scripts and Adobe Illustrator were used to generate better visualizations.

Animal experiments. Animal studies were conducted in compliance with animal protocols approved by the Institutional Animal Care and Use Committee of Sun Yat-sen University. Female BALB/c nude mice and NOD/SCID mice (5-6 weeks old) were purchased from the Beijing Vital River Laboratory Animal Technology Co. Tumor volume was measured by vernier caliper and calculated with the following formula: tumor volume $=$ width $^{2} \times$ length $\times 0.537$. Randomization was performed by equal division of tumor-bearing mice of similar tumor burden into different groups for drug treatment. For the SKOV3 tumor xenograft experiment, $4 \times 10^{6} \mathrm{SKOV} 3$ cells were injected s.c. in the right flank of the BALB/c nude mice. For PDX mouse models, PDX-POVC15 and PDXPOVC17 tumor masses were passaged to 40 NOD/SCID mice as mentioned above. When the tumors reached approximately $100 \mathrm{~mm}^{3}$, the mice were randomly divided into 4 groups for treatment: (a) vehicle; (b) trametinib; (c) LBH589; and (d) combination (trametinib and LBH589). Trametinib was dissolved in $0.5 \%$ methylcellulose and $0.2 \%$ Tween-80, and LBH589 was prepared in distilled $\mathrm{H}_{2} \mathrm{O}$ containing 2\% DMSO, 30\% PEG 300, and 5\% Tween-80. Drug dosages were given as follows: trametinib, $0.25 \mathrm{mg} / \mathrm{kg}$ daily, i.p.; and LBH589, $3.75 \mathrm{mg} / \mathrm{kg}$ every other day, i.p. PBS (1 $1 \times$ ) was used as the vehicle control. The tumor volumes and body weights were monitored 2-3 times per week until tumor volume reached $1000-1500 \mathrm{~mm}^{3}$. These mice were sacrificed by $\mathrm{CO}_{2}$ inhalation, and their tumors were harvested for further analysis.

Immunohistochemistry. IHC staining was performed using standard procedures. Briefly, xenograft tumors were harvested, fixed with formalin, and embedded in paraffin. After deparaffinization, rehydration, antigen retrieval by heat-induced epitope retrieval, and inactivation of endogenous peroxidase by $3 \% \mathrm{H}_{2} \mathrm{O}_{2}$, slides were blocked using a blocking solution and incubated overnight with primary antibodies. After incubation of secondary antibodies (Dako REAL HRP Rabbit/Mouse detection kit) for 30 minutes, the DAB reagent kit (ZSGB-BIO, ZLI9019) was used as chromogen and hematoxylin (ZSGB-BIO, ZLI-9609) as counterstain. Histoscore was a multiplicative index of the intensity of staining and the proportion of positive tumor cells. The intensity was graded as follows: 0 , negative staining; 1 , mild staining; 2, moderate staining; 3 , strong staining. The percentage of stained cells was defined as follows: 1 , less than $10 \% ; 2,10 \%-50 \% ; 3,50 \%-75 \%$; 4 , more than $75 \%$. Primary antibodies used were Ki67 (ZSGB-BIO, ZA-0502, sc-23900), p-ERK1/2 (Cell Signaling Technology 4370S), and DUSP6 (Abcam ab76310).

RNA interference and lentiviral infection. Cells were seeded into a 6-well plate before transfection with indicated siRNA. Transfection was performed using Lipofectamine RNAi-MAX (Thermo Fisher Scientific) following the manufacturer's instructions. The sequences of siRNAs are shown in Supplemental Table 3.

Human DUSP6 cDNA was subcloned into the NheI-BamHI restriction sites of the pCDH-CMV-MCS-EF1-copGFP lentiviral vector (MilliporeSigma). Human SPRY4 cDNA was subcloned into the NheI-BamHI restriction sites of the pCDH-EF1-MCS-T2A-puro lentiviral vector (MilliporeSigma). Viruses were generated by cotransfection of packaging vector psPAX2, envelope vector pMD2.G, and target plasmids into 293T cells using Lipo- 
fectamine 2000 (Thermo Fisher Scientific) according to the manufacturer's instructions. After 48 hours of transfection, virus-containing medium was harvested through a $0.45 \mu \mathrm{m}$ nylon filter and added to cell lines. Cell lines were sorted with GFP or puromycin for further analysis.

Data access. RNA-Seq and ChIP-Seq data were deposited in the NCBI's Sequence Read Archive (PRJNA698086).

Statistics. At least 3 biological replicates were performed for all experiments, and data are presented as mean \pm SD/SEM. All statistical analyses were performed using GraphPad Prism version 8.0, except for Fisher's exact test (SPSS version 24.0). Statistical differences were calculated using 2-tailed, unpaired Student's $t$ test, 1-way ANOVA with Bonferroni's post hoc test, and 2-way ANOVA with Tukey's post hoc test or Fisher's exact test. $P$ values less than 0.05 were considered statistically significant.

Study approval. All procedures involving the use and care of animals were conducted in compliance with standard procedures approved by the Institutional Animal Care and Use Committee of Sun Yat-sen University. The procedures to obtain all human material were approved by the medical ethics committee of the Sun Yat-sen University Cancer Center. Written informed consent was obtained from patients who provided the tissue.

\section{Author contributions}

SL, QZ, and JT conceived, designed, and supervised the study. SL and QZ performed the experiments. SL, XY, PG, and PD analyzed and interpreted the data (e.g., statistical analysis, biostatistics, computational analysis). JL, YX, H Lu, and WL provided patient samples. Jieping Chen provided pathology expertise. PD, XL, JY, Jinghong Chen, RC, ZY, RX, YS, JHH, H Liu, Jianfeng Chen, JB, JK, BW, and TK provided material and technical support. SL, QZ, and JT wrote the original draft of the manuscript. SL and JT reviewed and revised the manuscript. JYC, QY, and BTT provided a kind suggestion for the manuscript writing. All the authors gave their consent to publish this study.

\section{Acknowledgments}

We thank all the patients who donated samples for this study. We appreciate Dicong Zhu and Lizhi Liu for assisting the project. We thank Ting Xue for technical support. This work was supported by the National Natural Science Foundation of China (81972596, 81772963, and 81773279), the Guangdong Innovative and Entrepreneurial Research Team Program (2016ZT06S638, 2016ZT06S252), and the Sci-Tech Project Foundation of Guangzhou City (201707020039). The key raw data generated and/or analyzed during the current study are available at Research Data Deposit public platform (www.researchdata.org.cn) with approval number RDDB2021001636.

Address correspondence to: Jing Tan, State Key Laboratory of Oncology in South China, Collaborative Innovation Center for Cancer Medicine, Sun Yat-sen University Cancer Center, 651 East Dongfeng Road, Guangzhou, Guangdong 510060, China. Phone: 86.020.39336156; Email: tanjing@sysucc.org.cn.
1. Momenimovahed $\mathrm{Z}$, et al. Ovarian cancer in the world: epidemiology and risk factors. Int J Wom ens Health. 2019;11:287-299.

2. Banerjee S, Kaye SB. New strategies in the treatment of ovarian cancer: current clinical perspectives and future potential. Clin Cancer Res. 2013;19(5):961-968.

3. Holmes D. The problem with platinum. Nature. 2015;527(7579):S218-S219.

4. Kim JY, et al. Targeted therapy of ovarian cancer including immune check point inhibitor. Korean J Intern Med. 2017;32(5):798-804.

5. Geng R, et al. Synthetic lethal short hairpin RNA screening reveals that ring finger protein 183 confers resistance to trametinib in colorectal cancer cells. Chin J Cancer. 2017;36(1):63.

6. Jayson GC, et al. Ovarian cancer. Lancet. 2014;384(9951):1376-1388.

7. Yaeger R, Corcoran RB. Targeting alterations in the RAF-MEK pathway. Cancer Discov. 2019;9(3):329-341.

8. Cancer Genome Atlas Research Network. Integrated genomic analyses of ovarian carcinoma. Nature. 2011;474(7353):609-615.

9. Rissmann R, et al. Vemurafenib/dabrafenib and trametinib. Br JClin Pharmacol. 2015;80(4):765-767.

10. $\mathrm{Fu} \mathrm{H}$, et al. Aldolase A promotes proliferation and $\mathrm{G}_{1} / \mathrm{S}$ transition via the EGFR/MAPK pathway in non-small cell lung cancer. Cancer Commun (Lond). 2018;38(1):18.

11. Champer M, et al. Response to trametinib in recurrent low-grade serous ovarian cancer with NRAS mutation: a case report. Gynecol Oncol Rep. 2019;28:26-28.

12. Mert I, et al. Synergistic effect of MEK inhibitor and metformin combination in low grade serous ovarian cancer. Gynecol Oncol. 2017;146(2):319-326.

13. Gershenson DM, et al. A randomized phase II/ III study to assess the efficacy of trametinib in patients with recurrent or progressive low-grade serous ovarian or peritoneal cancer. Ann Oncol. 2019;30:v897-v898.

14. Farley J, et al. Selumetinib in women with recurrent low-grade serous carcinoma of the ovary or peritoneum: an open-label, single-arm, phase 2 study. Lancet Oncol. 2013;14(2):134-140.

15. Iavarone $\mathrm{C}$, et al. Combined MEK and BCL-2/ $\mathrm{X}_{\mathrm{L}}$ inhibition is effective in high-grade serous ovarian cancer patient-derived xenograft models and BIM levels are predictive of responsiveness. $\mathrm{Mol}$ Cancer Ther. 2019;18(3):642-655.

16. Gruosso T, et al. MAP3K8/TPL-2/COT is a potential predictive marker for MEK inhibitor treatment in high-grade serous ovarian carcinomas. Nat Commun. 2015;6:8583.

17. Cappuccio S, et al. Trametinib response in heavily pretreated high-grade ovarian cancer: one step towards precision medicine. Gynecol Oncol Rep. 2020;32:100547.

18. Coggins GE, et al. YAP1 mediates resistance to MEK1/2 inhibition in neuroblastomas with hyperactivated RAS signaling. Cancer Res. 2019;79(24):6204-6214.

19. Fedele C, et al. SHP2 inhibition prevents adaptive resistance to MEK inhibitors in multiple cancer models. Cancer Discov. 2018;8(10):1237-1249.

20. Emery CM, et al. MEK1 mutations confer resistance to MEK and B-RAF inhibition. Proc Natl Acad Sci U S A. 2009;106(48):20411-20416.

21. Hatzivassiliou G, et al. ERK inhibition overcomes acquired resistance to MEK inhibitors. Mol Cancer Ther. 2012;11(5):1143-1154.

22. Gao Y, et al. V211D mutation in MEK1 causes resistance to MEK inhibitors in colon cancer. Cancer Discov. 2019;9(9):1182-1191.

23. Little AS, et al. Amplification of the driving oncogene, KRAS or BRAF, underpins acquired resistance to MEK1/2 inhibitors in colorectal cancer cells. Sci Signal. 2011;4(166):ra17.

24. Ho AL, et al. Impact of combined mTOR and MEK inhibition in uveal melanoma is driven by tumor genotype. PLoS One. 2012;7(7):e40439.

25. Carson R, et al. HDAC inhibition overcomes acute resistance to MEK inhibition in BRAF-mutant colorectal cancer by downregulation of c-FLIPL. Clin Cancer Res. 2015;21(14):3230-3240.

26. Johnson GL, et al. Molecular pathways: adaptive kinome reprogramming in response to targeted inhibition of the BRAF-MEK-ERK pathway in cancer. Clin Cancer Res. 2014;20(10):2516-2522.

27. Friday BB, et al. BRAF V600E disrupts AZD6244-induced abrogation of negative feedback pathways between extracellular signal-regulated kinase and Raf proteins. Cancer Res. 2008;68(15):6145-6153.

28. Ambrosini $\mathrm{G}$, et al. Identification of unique MEK-dependent genes in GNAQ mutant uveal melanoma involved in cell growth, tumor cell invasion, and MEK resistance. Clin Cancer Res. 2012;18(13):3552-3561.

29. Forbes SA, et al. COSMIC: mining complete cancer genomes in the Catalogue of Somatic Mutations in Cancer. Nucleic Acids Res. 2011;39(database issue):D945-D950.

30. Barretina J, et al. The Cancer Cell Line Encyclope- 
dia enables predictive modelling of anticancer drug sensitivity. Nature. 2012;483(7391):603-607.

31. Beaufort CM, et al. Ovarian cancer cell line panel (OCCP): clinical importance of in vitro morphological subtypes. PLoS One. 2014;9(9):e103988.

32. Domcke S, et al. Evaluating cell lines as tumour models by comparison of genomic profiles. Nat Commun. 2013;4:2126.

33. Dry JR, et al. Transcriptional pathway signatures predict MEK addiction and response to selumetinib (AZD6244). Cancer Res. 2010;70(6):2264-2273.

34. Yao $X$, et al. VHL deficiency drives enhancer activation of oncogenes in clear cell renal cell carcinoma. Cancer Discov. 2017;7(11):1284-1305.

35. Heintzman ND, et al. Histone modifications at human enhancers reflect global cell-type-specific gene expression. Nature. 2009;459(7243):108-112.

36. Creyghton MP, et al. Histone H3K27ac separates active from poised enhancers and predicts developmental state. Proc Natl Acad Sci U S A. 2010;107(50):21931-21936.

37. Furukawa T, et al. Feedback regulation of DUSP6 transcription responding to MAPK1 via ETS2 in human cells. Biochem Biophys Res Commun. 2008;377(1):317-320.

38. Shojaee S, et al. Erk negative feedback control enables pre-B cell transformation and represents a therapeutic target in acute lymphoblastic leukemia. Cancer Cell. 2015;28(1):114-128.

39. Znosko WA, et al. Overlapping functions of Pea3 ETS transcription factors in FGF signaling during zebrafish development. Dev Biol. 2010;342(1):11-25.

40. Chen Z, et al. FGF signaling activates a Sox9Sox10 pathway for the formation and branching morphogenesis of mouse ocular glands. Development. 2014;141(13):2691-2701.

41. Qu X, et al. Glycosaminoglycan-dependent restriction of FGF diffusion is necessary for lacrimal gland development. Development. 2012;139(15):2730-2739.

42. Mason JM, et al. Sprouty proteins: multifaceted negative-feedback regulators of receptor tyrosine kinase signaling. Trends Cell Biol. 2006;16(1):45-54.

43. Sasaki A, et al. Mammalian Sprouty4 suppresses Ras-independent ERK activation by binding to Raf1. Nat Cell Biol. 2003;5(5):427-432.

44. Hrustanovic G, et al. RAS-MAPK dependence underlies a rational polytherapy strategy in EML4-ALK-positive lung cancer. Nat Med. 2015;21(9):1038-1047.

45. Shen $\mathrm{CH}$, et al. Loss of cohesin complex components STAG2 or STAG3 confers resistance to BRAF inhibition in melanoma. Nat Med. 2016;22(9):1056-1061.

46. Kitai H, et al. Epithelial-to-mesenchymal transition defines feedback activation of receptor tyrosine kinase signaling induced by MEK inhibition in KRAS-mutant lung cancer. Cancer Discov. 2016;6(7):754-769.

47. Lito P, et al. Relief of profound feedback inhibition of mitogenic signaling by RAF inhibitors attenuates their activity in BRAFV600E melanomas. Cancer Cell. 2012;22(5):668-682.

48. Day EK, et al. Glioblastoma cell resistance to EGFR and MET inhibition can be overcome via blockade of FGFR-SPRY2 bypass signaling. Cell Rep. 2020;30(10):3383-3396.

49. Easwaran H, et al. Cancer epigenetics: tumor heterogeneity, plasticity of stem-like states, and drug resistance. Mol Cell. 2014;54(5):716-727.

50. Sharma SV, et al. A chromatin-mediated reversible drug-tolerant state in cancer cell subpopulations. Cell. 2010;141(1):69-80.

51. Zawistowski JS, et al. Enhancer remodeling during adaptive bypass to MEK inhibition is attenuated by pharmacologic targeting of the P-TEFb complex. Cancer Discov. 2017;7(3):302-321

52. Jing J, et al. Comprehensive predictive biomarker analysis for MEK inhibitor GSK1120212. Mol Cancer Ther. 2012;11(3):720-729.

53. Li F, et al. FGFR-mediated reactivation of MAPK signaling attenuates antitumor effects of imatinib in gastrointestinal stromal tumors. Cancer Discov. 2015;5(4):438-451.

54. Lai AZ, et al. Dynamic reprogramming of signaling upon met inhibition reveals a mechanism of drug resistance in gastric cancer. Sci Signal. 2014;7(322):ra38.

55 . Malchers F, et al. Mechanisms of primary drug resistance in FGFR1-amplified lung cancer. Clin Cancer Res. 2017;23(18):5527-5536.

56. Yamada T, et al. Histone deacetylase inhibition enhances the antitumor activity of a MEK inhibitor in lung cancer cells harboring RAS mutations. Mol Cancer Ther. 2018;17(1):17-25.

57. Peng DH, et al. ZEB1 suppression sensitizes KRAS mutant cancers to MEK inhibition by an IL17RD-dependent mechanism. Sci Transl Med. 2019;11(483):eaaq1238.

58. Booth L, et al. The HDAC inhibitor AR42 interacts with pazopanib to kill trametinib/dabrafenib-resistant melanoma cells in vitro and in vivo. Oncotarget. 2017;8(10):16367-16386.

59. Faiao-Flores F, et al. HDAC inhibition enhances the in vivo efficacy of MEK inhibitor therapy in uveal melanoma. Clin Cancer Res. 2019;25(18):5686-5701.

60. Tan J, et al. PDK1 signaling toward PLK1-MYC activation confers oncogenic transformation, tumor-initiating cell activation, and resistance to mTOR-targeted therapy. Cancer Discov. 2013;3(10):1156-1171. 61. Chen S, et al. fastp: an ultra-fast all-in-one
FASTQ preprocessor. Bioinformatics. 2018;34(17):i884-i890.

62. Dobin A, et al. STAR: ultrafast universal RNA-seq aligner. Bioinformatics. 2013;29(1):15-21.

63. Li B, Dewey CN. RSEM: accurate transcript quantification from RNA-Seq data with or without a reference genome. BMC Bioinformatics. 2011;12:323.

64. Love MI, et al. Moderated estimation of fold change and dispersion for RNA-seq data with DESeq2. Genome Biol. 2014;15(12):550.

65. Subramanian A, et al. Gene set enrichment analysis: a knowledge-based approach for interpreting genome-wide expression profiles. Proc Natl Acad Sci U S A. 2005;102(43):15545-15550.

66. Ward CM, et al. ngsReports: a Bioconductor package for managing FastQC reports and other NGS related $\log$ files. Bioinformatics. 2019;36(8):2587-2588.

67. Li H, Durbin R. Fast and accurate short read alignment with Burrows-Wheeler transform. Bioinformatics. 2009;25(14):1754-1760.

68. Li H, et al. The sequence alignment/map format and SAMtools. Bioinformatics. 2009;25(16):2078-2079.

69. Feng J, et al. Identifying ChIP-seq enrichment using MACS. Nat Protoc. 2012;7(9):1728-1740.

70. Zhang Y, et al. Model-based analysis of ChIP-Seq (MACS). Genome Biol. 2008;9(9):R137.

71. Amemiya HM, et al. The ENCODE blacklist: identification of problematic regions of the genome. Sci Rep. 2019;9(1):9354.

72. Yu G, et al. ChIPseeker: an R/Bioconductor package for ChIP peak annotation, comparison and visualization. Bioinformatics. 2015;31(14):2382-2383.

73. Shen L, et al. ngs.plot: quick mining and visualization of next-generation sequencing data by integrating genomic databases. BMC Genomics. 2014;15:284.

74. Abugessaisa I, et al. refTSS: a reference data set for human and mouse transcription start sites. J Mol Biol. 2019;431(13):2407-2422.

75. Neph S, et al. BEDOPS: high-performance genomic feature operations. Bioinformatics. 2012;28(14):1919-1920.

76. Quinlan AR, Hall IM. BEDTools: a flexible suite of utilities for comparing genomic features. Bioinformatics. 2010;26(6):841-842.

77. McLean CY, et al. GREAT improves functional interpretation of cis-regulatory regions. Nat Biotechnol. 2010;28(5):495-501.

78. Anders S, et al. HTSeq-a Python framework to work with high-throughput sequencing data. Bioinformatics. 2015;31(2):166-169.

79. Ramirez F, et al. deepTools2: a next generation web server for deep-sequencing data analysis. Nucleic Acids Res. 2016;44(w1):W160-W165. 